\title{
Convergence analysis of finite element method for a parabolic obstacle problem
}

\author{
Thirupathi Gudi*, Papri Majumder \\ Department of Mathematics, Indian Institute of Science, Bangalore 560012, India
}

\section{A R T I C L E I N F O}

\section{Article history:}

Received 23 February 2017

Received in revised form 3 July 2018

\section{MSC:}

$65 \mathrm{~N} 30$

$65 \mathrm{~N} 15$

Keywords:

Finite element

Parabolic obstacle problem

Variational inequalities

\begin{abstract}
A B S T R A C T
A conforming finite element method is proposed and analyzed for numerical approximation of the solution of a parabolic variational inequality of the obstacle problem. The model problem, which is originally proposed using a general obstacle, is reframed as a model problem with zero obstacle but with non-homogeneous Dirichlet boundary conditions. Subsequently the discrete problem is reframed and the error analysis proving the convergence of the method is performed. The analysis requires a positive preserving interpolation with non-homogeneous Dirichlet boundary condition and a post-processed solution that satisfies the boundary conditions sharply. The results in the article extend the results of (Johnson, SINUM, 1976) for a zero obstacle function to a more general obstacle function.
\end{abstract}

(C) 2019 Elsevier B.V. All rights reserved.

\section{Introduction}

The numerical analysis of parabolic variational inequalities has been an active field of research in the past few decades. The parabolic obstacle problem is one of the well known typical models for studying the parabolic variational inequalities. The parabolic obstacle problem appears naturally in the American option problem, Stefan problem and in electrochemical machining problem. The numerical analysis of this class of problems has created interest as they naturally provide challenges both in theory and computation. Some studies dealing with error analysis for finite element approximations of parabolic variational inequalities have successfully been done in the literature [1-8]. In [2], the convergence of a truncation method for the numerical solution of the parabolic variational inequality has been obtained with obstacle $\psi \in C^{2}(\bar{\Omega})$ and $\psi \leq 0$ on the boundary $\partial \Omega$, under the assumptions $f, f_{t} \in C([0, T] ; \bar{\Omega})$, where $f$ is the source term. An $L^{\infty}$-convergence of an approximation for the parabolic variational inequality with the zero obstacle has been established under the regularity condition $u_{t t} \in L^{2}\left([0, T] ; L^{2}(\Omega)\right)$ and with a certain assumption on the angle of the triangle $T$ in triangulation $\mathcal{T}_{h}$ in [3]. An $L^{2}$-error estimate for a fully-discrete approximation of the solution of parabolic variational inequality with the zero obstacle has been studied in [4]. In that article the fully-discrete scheme is defined by continuous linear finite element for space approximation and general $\theta$-scheme (i.e. a general finite difference discretization in time including the backward Euler, forward Euler and Crank-Nicolson scheme) for time discretization. An a posteriori error analysis for parabolic variational inequalities (motivated by American option for baskets) has been done in [5]. In that article the authors gave an upper bound for the error in $L^{2}\left(0, T ; H^{1}(\Omega)\right)$ of fully discrete method (i.e. piecewise linear finite elements in space and the backward Euler method in time). In [6] the authors studied a finite element approximation of a parabolic obstacle problem with nonsmooth initial data for one dimension of spatial variable. An error estimate for

\footnotetext{
* Corresponding author.

E-mail addresses: gudi@iisc.ac.in (T. Gudi), paprim@iisc.ac.in (P. Majumder).
} 
parabolic variational inequalities in the uniform norm has been derived in [7]. In that article, the authors coupled the finite element spatial approximation with a semi-implicit scheme to give $L^{\infty}$-asymptotic behavior in the uniform norm using discrete maximum principles. In [8], the authors dealt with the design and analysis of a finite element approximation of the parabolic fractional obstacle problem in bounded domains. This analysis relies on the construction and approximation properties of a positivity preserving interpolant. They discretized the truncation with a backward Euler scheme in time, and use first-degree tensor product finite elements for space. Some error estimations dealing with parabolic variational inequality arising from American option problem, Stefan problem, fluid problem, etc. have been studied in [9-15]. For an example, in [9] the authors established an error estimate for finite element approximations of American option prices under admissible regularity. In [13] the authors derived a priori error estimate for Stefan problem. For the theory of variational inequalities and their corresponding numerical analysis, we refer to the books [16-23]. Moreover, some related numerical analysis for elliptic, parabolic variational inequalities, PDEs and their solvers may be found in [24-26]. For an example, in [26] the authors derived a posteriori error estimate for implicit backward Euler approximation of parabolic PDEs in Hilbert space.

In this article, we study the convergence analysis of a finite element method for parabolic obstacle problem with general obstacle, i.e., with obstacle $\psi \in H^{2}(\Omega)$ and $\left.\psi\right|_{\partial \Omega} \leq 0$ on boundary $\partial \Omega$, where $\Omega \subset \mathbb{R}^{2}$. Here, actually we generalize the analysis in [1]. More explicitly, the results in the article can be described as follows since the generalization of the analysis in [1] to the model problem (2.1)-(2.2) is not straight forward:

- In [1], the model problem is with zero obstacle function. Even though, the analysis for that case was quite difficult, the generalization to nonzero obstacle requires some more attention. For example, let $\tilde{\mathcal{K}}_{h}$ be the discrete version of $\tilde{\mathcal{K}}$ defined by interpolating the obstacle at the mesh points. Then the interpolation defined in [1] does not preserve $\tilde{\mathcal{K}}_{h} \subset \tilde{\mathcal{K}}$.

- Secondly since $\partial u / \partial t$ is not a continuous function, where $u$ is the exact solution, we cannot use the Lagrange interpolation. Because the error analysis requires the time derivative and interpolation commute. In [1], Johnson used a different approach for defining the interpolation that is valid for $H^{1}(\Omega)$ functions and at the same time it preserves $\tilde{\mathcal{K}}_{h} \subset \tilde{\mathcal{K}}$ (when $\psi$ is identically zero) and further the interpolation of $u$ therein is an element of $\tilde{\mathcal{K}}_{h}$ when the obstacle is a zero function.

- In this article, we have non-zero obstacle, therefore the additional difficulties arise due to the nonconforming approximation $\tilde{\mathcal{K}}_{h} \not \subset \tilde{\mathcal{K}}$. We rewrite the original problem into a problem with zero obstacle by translation and by introducing nonhomogeneous Dirichlet boundary condition. Then we can use the positivity preserving interpolation (with appropriate modifications at the boundary) in [27,28].

- In [1], the analysis uses the discrete solution to be in $\mathcal{K}$. But since the nonhomogeneous Dirichlet boundary condition are approximated on the boundary, this cannot hold. We require to define a post-processed solution satisfying the Dirichlet boundary conditions sharply from the discrete solution.

The rest of the article is organized as follows. In Section 2, we first introduce some notations and give the continuous setting of the obstacle problem with general obstacle. Then we convert the general obstacle problem into zero obstacle problem with non homogeneous boundary condition by translation. We recall some required known regularity results from [29]. In Section 3, we define the discrete problem and define an interpolation operator which satisfies a stability property in $L^{2}$ and $H^{1}$. We then derive corresponding interpolation error estimates. Further, we construct a post-processed discrete solution, and derive some error estimate for the discrete solution and post-processed solution. In Section 4, we discuss a priori error estimates with these ingredients and derive order of convergence $h+(\Delta t)^{\frac{3}{4}}\left(\log \frac{1}{\Delta t}\right)^{\frac{1}{4}}$. Finally in Section 5 , we present numerical experiments to illustrate the theoretical results.

\section{Model problem}

Let $\Omega \subset \mathbb{R}^{2}$ be a convex polygonal domain with boundary $\partial \Omega$. We postpone the 3 dimensional case to future research as there will be some technical difficulties in the analysis, although the ideas could be similar. For a nonnegative integer $m \geq 0$ and $1 \leq p \leq \infty$, let $W^{m, p}(\Omega)$ be the Sobolev space equipped with the norm

$$
\|v\|_{W^{m, p}(\Omega)}:=\left(\sum_{|\alpha| \leq m} \int_{\Omega}\left|D^{\alpha} v\right|^{p} d x\right)^{1 / p},
$$

with the standard modification for $p=\infty$. Here $D^{\alpha}$ denotes the distribution derivative of order $|\alpha|$. We denote the space by $H^{m}(\Omega)$ when $p=2$. The space $H_{0}^{1}(\Omega)$ denotes the subspace of $H^{1}(\Omega)$ with zero trace (vanishing on the boundary in the trace sense).

Let $\mathcal{J}=[0, T](T>0)$ be the time interval that the problem is defined. If $X$ is a normed linear space equipped with the norm $\|\cdot\|_{X}$, then define the spaces $L^{p}(\mathcal{J} ; X)$ for $1 \leq p \leq \infty$ as the set of all functions $w: \mathcal{J} \rightarrow X$ such that

$$
\|w\|_{L^{p}(\mathcal{J} ; X)}:=\left(\int_{0}^{T}\|w(t)\|_{X}^{p} d t\right)^{1 / p}, \quad 1 \leq p<\infty
$$


and

$$
\|w\|_{L^{\infty}(\mathcal{J} ; X)}:=\sup _{t \in \mathcal{J}}\|w(t)\|_{X} .
$$

Finally let $C(\mathcal{J} ; X)$ be the space of all continuous functions $w: \mathcal{J} \rightarrow X$.

Define a bilinear form $a$ by $a(w, v)=(\nabla w, \nabla v)$. Hereafter, we denote the $\left[L^{2}(\Omega)\right]^{d}$ inner product by $(\cdot, \cdot)$, where $d=1,2$.

Let $\psi \in W^{2, \infty}(\Omega)$ be a given function with $\left.\psi\right|_{\partial \Omega} \leq 0$. Define the closed convex set by

$$
\tilde{\mathcal{K}}:=\left\{v \in H_{0}^{1}(\Omega): v \geq \psi \text { a.e. in } \Omega\right\} .
$$

The model problem consists of finding $u: \mathcal{J} \rightarrow \tilde{\mathcal{K}}$ such that a.e. on $\mathcal{J}$,

$$
\begin{aligned}
\left(\frac{\partial u}{\partial t}, v-u\right)+a(u, v-u) & \geq(f, v-u) \quad \forall v \in \tilde{\mathcal{K}}, \\
u(x, 0) & =u_{0}(x) \quad x \in \Omega,
\end{aligned}
$$

where

$$
\begin{aligned}
& f \in C\left(\mathcal{J} ; L^{\infty}(\Omega)\right), \quad \frac{\partial f}{\partial t} \in L^{2}\left(\mathcal{J} ; L^{\infty}(\Omega)\right), \\
& u_{0} \in W^{2, \infty}(\Omega) \cap \tilde{\mathcal{K}} .
\end{aligned}
$$

The model problem (2.1)-(2.2) has a unique solution $u$ and there holds [29]

$$
\begin{aligned}
& u \in L^{\infty}\left(\mathcal{J} ; W^{2, p}(\Omega)\right), \quad 1 \leq p<\infty, \\
& \frac{\partial u}{\partial t} \in L^{2}\left(\mathcal{J} ; H_{0}^{1}(\Omega)\right) \cap L^{\infty}\left(\mathcal{J} ; L^{\infty}(\Omega)\right), \\
& \left(\frac{\partial^{+} u}{\partial t}, v-u\right)+a(u, v-u) \geq(f, v-u) \quad \forall v \in \tilde{\mathcal{K}}, t \in \mathcal{J},
\end{aligned}
$$

where $\partial^{+} u / \partial t$ denotes the right-hand derivative of $u$ with respect to $t$. Define the set

$$
\mathcal{K}:=\left\{v \in H^{1}(\Omega): v \geq 0 \text { a.e. in } \Omega,\left.\quad v\right|_{\partial \Omega}=-\psi\right\} .
$$

We introduce $w:=u-\psi$. Then $w$ satisfies $w: \mathcal{J} \rightarrow \mathcal{K}$ and

$$
\begin{aligned}
\left(\frac{\partial w}{\partial t}, v-w\right)+a(w, v-w) & \geq(f+\Delta \psi, v-w) \quad \forall v \in \mathcal{K}, \text { a.e. on } \mathcal{J}, \\
w(x, 0) & =u_{0}(x)-\psi(x) \quad x \in \Omega .
\end{aligned}
$$

It is immediate to see that the model problem (2.4)-(2.5) has a unique solution $w$ and there holds

$$
\begin{aligned}
& w \in L^{\infty}\left(\mathcal{J} ; W^{2, p}(\Omega)\right), \quad 1 \leq p<\infty, \\
& \frac{\partial w}{\partial t} \in L^{2}\left(\mathcal{J} ; H_{0}^{1}(\Omega)\right) \cap L^{\infty}\left(\mathcal{J} ; L^{\infty}(\Omega)\right), \\
& \left(\frac{\partial^{+} w}{\partial t}, v-w\right)+a(w, v-w) \geq(f+\Delta \psi, v-w) \quad \forall v \in \mathcal{K}, t \in \mathcal{J},
\end{aligned}
$$

where $\partial^{+} w / \partial t$ denotes the right-hand derivative of $w$ with respect to $t$. Since the solution $u$ of (2.1)-(2.2) satisfies for all $t \in \mathcal{J}$ (see [29]) that

$$
\begin{aligned}
& \frac{\partial^{+} u}{\partial t}=\Delta u+f \quad \text { a.e. on } \Omega^{+}(t), \\
& \frac{\partial^{+} u}{\partial t}=\max \{f+\Delta \psi, 0\} \quad \text { a.e. on } \Omega^{0}(t),
\end{aligned}
$$

where $\Omega^{+}(t):=\{x \in \Omega: u(x, t)>\psi(x)\}$ and $\Omega^{0}(t):=\{x \in \Omega: u(x, t)=\psi(x)\}$. We conclude that the solution $w$ of (2.4)-(2.5) satisfies

$$
\begin{aligned}
& \frac{\partial^{+} w}{\partial t}=\Delta w+f+\Delta \psi \quad \text { a.e. on } \Omega^{+}(t), \\
& \frac{\partial^{+} w}{\partial t}=\max \{f+\Delta \psi, 0\} \quad \text { a.e. on } \Omega^{0}(t),
\end{aligned}
$$

where $\Omega^{+}(t):=\{x \in \Omega: w(x, t)>0\}$ and $\Omega^{0}(t):=\{x \in \Omega: w(x, t)=0\}$. 


\section{Discrete problem}

Let $\mathcal{T}_{h}$ be a regular simplicial triangulation of $\Omega$. Denote the set of all vertices of $\mathcal{T}_{h}$ that are in $\Omega$ (resp. on $\partial \Omega$ ) by $\mathcal{V}_{h}^{i}$ (resp. $\mathcal{V}_{h}^{b}$ ). Set $\mathcal{V}_{h}=\mathcal{V}_{h}^{i} \cup \mathcal{V}_{h}^{b}$. Similarly, denote the set of all interior edges of $\mathcal{T}_{h}$ by $\mathcal{E}_{h}^{i}$, the set of all boundary edges by $\mathcal{E}_{h}^{b}$, and define $\mathcal{E}_{h}=\mathcal{E}_{h}^{i} \cup \mathcal{E}_{h}^{b}$. Let $h_{T}$ be the diameter of $T$, where $T$ is a triangle of $\mathcal{T}_{h}$. Set $h:=\max \left\{h_{T}: T \in \mathcal{T}_{h}\right\}$ and $|T|:=$ area of $T$. The set of three vertices of $T$ is denoted by $\mathcal{V}_{T}$ and the length of an edge $e \in \mathcal{E}_{h}$ is denoted by $h_{e}$. Here each triangle $T$ in $\mathcal{T}_{h}$ is assumed to be closed. Define $\mathcal{T}_{h}^{i}$ to be the set of all triangles which do not share an edge with boundary $\partial \Omega$. Let $\mathcal{T}_{h}^{b}$ denote the remaining set of triangles, i.e., the set of all triangles $T \in \mathcal{T}_{h}$ which have an edge $e \in \mathcal{E}_{h}^{b}$ on its boundary $\partial T$. For simplicity assume that any $T \in \mathcal{T}_{h}^{b}$ shares at most one edge with boundary $\partial \Omega$, otherwise the triangle $T$ has no interior node.

Define the discrete space

$$
V_{h}:=\left\{v_{h} \in C(\bar{\Omega}):\left.v_{h}\right|_{T} \in \mathbb{P}_{1}(T) \quad \forall T \in \mathcal{T}_{h}\right\},
$$

where $\mathbb{P}_{r}(D)$ is the set of all polynomials of degree at most $r$ and defined on an open set $D \subset \Omega$. Define

$$
\mathcal{K}_{h}:=\left\{v_{h} \in V_{h}: v_{h}(z) \geq 0, \quad \forall z \in \mathcal{V}_{h}^{i} ; \quad v_{h}(z)=-\psi(z), \quad \forall z \in \mathcal{V}_{h}^{b}\right\} .
$$

For some positive integer $N$, let $0=t_{0}<t_{1}<t_{2}<\cdots<t_{N-1}<t_{N}=\mathrm{T}$ be a uniform partition of $\mathcal{J}=[0, \mathrm{~T}]$ with $\Delta t=\mathrm{T} / N$, and let $\mathcal{J}_{n}=\left(t_{n-1}, t_{n}\right]$. On a discrete set $\left\{v^{0}, v^{1}, v^{2}, \ldots, v^{N}\right\}$ of $N+1$ points, define

$$
\partial v^{n}=\frac{v^{n}-v^{n-1}}{\Delta t} \text { for } n=1,2, \ldots, N .
$$

The fully discrete finite element method for (2.4)-(2.5) is defined as to find $W_{h}^{n} \in \mathcal{K}_{h}$ for $n \geq 1$ such that

$$
\begin{aligned}
\left(\partial W_{h}^{n}, v_{h}-W_{h}^{n}\right)+a\left(W_{h}^{n}, v_{h}-W_{h}^{n}\right) & \geq\left(f\left(t_{n}\right)+\Delta \psi, v_{h}-W_{h}^{n}\right) \quad \forall v_{h} \in \mathcal{K}_{h}, \\
W_{h}^{0} & =\pi_{h}\left(u_{0}-\psi\right) \in \mathcal{K}_{h},
\end{aligned}
$$

where $\pi_{h}$ is an appropriate interpolation operator.

The following assumption is made as in [1]:

If $D_{n}=\cup_{t \in \mathcal{J}_{n}}\left(\Omega^{+}(t) \cup \Omega^{+}\left(t_{n}\right)\right) \backslash \overline{\left(\Omega^{+}(t) \cap \Omega^{+}\left(t_{n}\right)\right)}$, then there holds for constant $C$

$$
\sum_{n=1}^{N} m\left(D_{n}\right) \leq C,
$$

where $m(D)$ is the Lebesgue measure of $D \subset \mathbb{R}^{2}$.

Note: Hereafter, $\mathrm{C}$ will denote a positive constant, not necessarily the same at each appearance, which is independent of the parameters $\Delta t$ and $h$.

\subsection{Positive preserving interpolation}

In this section we modify slightly the positive preserving interpolation operator introduced in [27], to deal with nonhomogeneous Dirichlet boundary conditions. Let $v \in H^{1}(\Omega)$ be such that $\left.v\right|_{\partial \Omega}=g \in C(\partial \Omega)$. For any $z \in \mathcal{V}_{h}^{i}$, let $\omega_{z}$ be the patch of the vertex $z$ which is the union of all the triangles sharing $z$. Define $\Delta_{z}$ as the largest disk that can be inscribed in $\omega_{z}$ with center at $z$. Then, define $\alpha_{z} \in \mathbb{R}$ by

$$
\alpha_{z}:= \begin{cases}\frac{1}{\left|\Delta_{z}\right|} \int_{\Delta_{z}} v(x) d x & \text { if } \quad z \in \mathcal{V}_{h}^{i}, \\ v(z) & \text { if } \quad z \in \mathcal{V}_{h}^{b},\end{cases}
$$

where $\left|\Delta_{z}\right|$ is the two dimensional Lebesgue measure of the disk $\Delta_{z}$. Then define the interpolation $\pi_{h} v \in V_{h}$ by

$$
\pi_{h} v(x):=\sum_{z \in \mathcal{V}_{h}} \alpha_{z} \phi_{z}(x)
$$

where $\phi_{z} \in V_{h}$ is the Lagrange basis function associated to the vertex $z$ satisfying

$$
\phi_{z_{i}}\left(z_{j}\right)=\delta_{i j} \text { for } 1 \leq i, j \leq \operatorname{dim}\left(V_{h}\right),
$$

and $\delta_{i j}$ is the Kronecker delta function. Note that if $v \in \mathcal{K}$, then $\pi_{h} v \in \mathcal{K}_{h}$. For any $T \in \mathcal{T}_{h}$, define

$$
S_{T}:=\cup_{z \in \mathcal{V}_{T}} \omega_{z} .
$$

If all three vertices of $T$ are inside $\Omega$ and $v \in \mathbb{P}_{1}\left(S_{T}\right)$, then it is easy to check that $\pi_{h} v \equiv v$ on $T$, see [27]. Further if at least one vertex is on $\partial \Omega$ and $v \in \mathbb{P}_{1}\left(S_{T}\right)$, we have again $\pi_{h} v \equiv v$ on $T$. We prove the stability estimates for $\pi_{h}$. 
Lemma 3.1. Let $v \in H^{1}(\Omega)$ be such that $\left.v\right|_{\partial \Omega}=g \in H^{3 / 2}(\partial \Omega)$. If $T \in \mathcal{T}_{h}$ with $T \cap \partial \Omega=\emptyset$, then

$$
\begin{aligned}
\left\|\pi_{h} v\right\|_{L^{2}(T)} & \leq C\|v\|_{L^{2}\left(S_{T}\right)}, \\
\left\|\nabla \pi_{h} v\right\|_{L^{2}(T)} & \leq C\|\nabla v\|_{L^{2}\left(S_{T}\right)} .
\end{aligned}
$$

Further if $T \in \mathcal{T}_{h}$ with $T \cap \partial \Omega \neq \emptyset$, then

$$
\begin{aligned}
\left\|\pi_{h} v\right\|_{L^{2}(T)} & \leq C\left(\|v\|_{L^{2}\left(S_{T}\right)}+h_{T} \max _{z \in \mathcal{V}_{T} \cap \mathcal{V}_{h}^{b}}|g(z)|\right), \\
\left\|\pi_{h} v\right\|_{L^{2}(T)} & \leq C\left(\|v\|_{L^{2}\left(S_{T}\right)}+h_{T}\|\nabla v\|_{L^{2}\left(S_{T}\right)}+h_{T}^{3 / 2}\left\|g^{\prime}\right\|_{L^{2}\left(\partial S_{T} \cap \partial \Omega\right)}\right) \\
\left\|\nabla \pi_{h} v\right\|_{L^{2}(T)} & \leq C\left(\|\nabla v\|_{L^{2}\left(S_{T}\right)}+h_{T}^{1 / 2}\left\|g^{\prime}\right\|_{L^{2}\left(\partial S_{T} \cap \partial \Omega\right)}\right) .
\end{aligned}
$$

Proof. We present the proof in several steps.

Proof of (3.4) and (3.6): Note that if $z \in \mathcal{V}_{h}^{i}$, then it is easy to show that

$$
\left|\alpha_{z}\right| \leq C h_{T}^{-1}\|v\|_{L^{2}\left(S_{T}\right)},
$$

and if $z \in \mathcal{V}_{h}^{b}$, then it is clear that

$$
\left|\alpha_{z}\right|=|g(z)|
$$

Since

$$
\left\|\pi_{h} v\right\|_{L^{2}(T)}^{2} \leq C \sum_{z \in \mathcal{V}_{T}}\left|\alpha_{z}\right|^{2}\left\|\phi_{z}\right\|_{L^{2}(T)}^{2} \leq C \sum_{z \in \mathcal{V}_{T}} h_{T}^{2}\left|\alpha_{z}\right|^{2},
$$

the proof of $L^{2}$-stability in (3.4) and (3.6) follows.

Proof of (3.5): Since $T \in \mathcal{T}_{h}$ with $T \cap \partial \Omega=\emptyset$, we have that all $z \in \mathcal{V}_{T}$ are inside of $\Omega$. Then note that for any $c \in \mathbb{R}$, $\pi_{h} c=c$ on $T$ and

$$
\begin{aligned}
\left\|\nabla \pi_{h} v\right\|_{L^{2}(T)} & =\left\|\nabla\left(\pi_{h} v+c\right)\right\|_{L^{2}(T)}=\left\|\nabla \pi_{h}(v+c)\right\|_{L^{2}(T)} \leq C h_{T}^{-1}\left\|\pi_{h}(v+c)\right\|_{L^{2}(T)} \\
& \leq C h_{T}^{-1}\|v+c\|_{L^{2}\left(S_{T}\right)},
\end{aligned}
$$

where we have used (3.4) in the last step. Now choose $c$ as the integral mean of $v$ on $S_{T}$ and use scaling argument to derive

$$
\left\|\nabla \pi_{h} v\right\|_{L^{2}(T)} \leq C\|\nabla v\|_{L^{2}\left(S_{T}\right)} .
$$

This proves (3.5).

Proof of (3.7): Now suppose that there is some $z \in \mathcal{V}_{T}$ such that $z \in \mathcal{V}_{h}^{b}$.

Case (i): Let $\partial \Omega \cap \partial T$ be an edge and denote it by $e$. Since $g \in C(e)$, there is some $z_{0} \in e$ such that

$$
g\left(z_{0}\right)=\frac{1}{h_{e}} \int_{e} g(s) d s .
$$

Note that

$$
\left|g\left(z_{0}\right)\right|=h_{e}^{-1 / 2}\|g\|_{L^{2}(e)}=h_{e}^{-1 / 2}\|v\|_{L^{2}(e)},
$$

and since $g \in H^{1}(e)$, we have that $g$ is absolutely continuous on $e$. Now for any $z \in e$,

$$
|g(z)| \leq\left|g\left(z_{0}\right)\right|+h_{e}^{1 / 2}\left\|g^{\prime}\right\|_{L^{2}(e)},
$$

where $g^{\prime}$ denotes the tangential derivative of $g$ on $e$. By the trace inequality

$$
\begin{aligned}
|g(z)| & \leq h_{e}^{-1 / 2}\|v\|_{L^{2}(e)}+h_{e}^{1 / 2}\left\|g^{\prime}\right\|_{L^{2}(e)} \\
& \leq C\left(h_{e}^{-1}\|v\|_{L^{2}(T)}+\|\nabla v\|_{L^{2}(T)}\right)+h_{e}^{1 / 2}\left\|g^{\prime}\right\|_{L^{2}(e)} .
\end{aligned}
$$

Therefore using this in (3.6), we find

$$
\begin{aligned}
\left\|\pi_{h} v\right\|_{L^{2}(T)} & \leq C\left(\|v\|_{L^{2}\left(S_{T}\right)}+\|v\|_{L^{2}(T)}+h_{T}\|\nabla v\|_{L^{2}(T)}+h_{T}^{3 / 2}\left\|g^{\prime}\right\|_{L^{2}(e)}\right) \\
& \leq C\left(\|v\|_{L^{2}\left(S_{T}\right)}+h_{T}\|\nabla v\|_{L^{2}(T)}+h_{T}^{3 / 2}\left\|g^{\prime}\right\|_{L^{2}(e)}\right) .
\end{aligned}
$$


Case (ii): If $\partial \Omega \cap \partial T$ is not an edge, then choose an edge $e \in \mathcal{E}_{h}^{b}$ such that $z \in e$. Let $\tilde{T} \in \mathcal{T}_{h}$ be a triangle with $e \subset \partial \tilde{T}$. Repeating the arguments that are used for proving (3.9), we arrive at

$$
|g(z)| \leq C\left(h_{e}^{-1}\|v\|_{L^{2}(\tilde{T})}+\|\nabla v\|_{L^{2}(\tilde{T})}\right)+h_{e}^{1 / 2}\left\|g^{\prime}\right\|_{L^{2}(e)} .
$$

Since $\tilde{T} \subset S_{T}$ and $e \subset \partial S_{T} \cap \partial \Omega$, we have

$$
|g(z)| \leq C\left(h_{e}^{-1}\|v\|_{L^{2}\left(S_{T}\right)}+\|\nabla v\|_{L^{2}\left(S_{T}\right)}\right)+h_{e}^{1 / 2}\left\|g^{\prime}\right\|_{L^{2}\left(\partial S_{T} \cap \partial \Omega\right)} .
$$

Substituting the estimate for $g(z)$ in (3.6), we obtain (3.7).

Proof of (3.8): For the estimate on $\nabla \pi_{h} v$, consider any $c \in \mathbb{R}$ and

$$
\begin{aligned}
\left\|\nabla \pi_{h} v\right\|_{L^{2}(T)} & =\left\|\nabla \pi_{h}(v-c)\right\|_{L^{2}(T)} \leq C h_{T}^{-1}\left\|\pi_{h}(v-c)\right\|_{L^{2}(T)} \\
& \leq C h_{T}^{-1}\left(\|v-c\|_{L^{2}\left(S_{T}\right)}+h_{T}\|\nabla v\|_{L^{2}(T)}+h_{T}^{3 / 2}\left\|g^{\prime}\right\|_{L^{2}\left(\partial S_{T} \cap \partial \Omega\right)}\right),
\end{aligned}
$$

where we have used (3.7) in the last step. Again choosing $c$ as the integral mean of $v$ on $S_{T}$ and using scaling argument, we find that

$$
\left\|\nabla \pi_{h} v\right\|_{L^{2}(T)} \leq C\left(\|\nabla v\|_{L^{2}\left(S_{T}\right)}+h_{T}^{1 / 2}\left\|g^{\prime}\right\|_{L^{2}\left(\partial S_{T} \cap \partial \Omega\right)}\right) .
$$

Therefore the proof follows.

The following approximation properties now follow from the stability and polynomial invariance of $\pi_{h}$ :

Theorem 3.2. Let $v \in H^{m+1}(\Omega)(0 \leq m \leq 1)$ be such that $\left.v\right|_{\partial \Omega}=g \in H^{3 / 2}(\partial \Omega)$. If $T \in \mathcal{T}_{h}$ with $T \cap \partial \Omega=\emptyset$, then

$$
\begin{aligned}
\left\|v-\pi_{h} v\right\|_{L^{2}(T)} & \leq C h_{T}^{m+1}\|v\|_{H^{m+1}\left(S_{T}\right)}, \\
\left\|\nabla\left(v-\pi_{h} v\right)\right\|_{L^{2}(T)} & \leq C h_{T}^{m}\|v\|_{H^{m+1}\left(S_{T}\right)} .
\end{aligned}
$$

Further if $T \in \mathcal{T}_{h}$ with $T \cap \partial \Omega \neq \emptyset$, then

$$
\begin{aligned}
\left\|v-\pi_{h} v\right\|_{L^{2}(T)} & \leq C\left(h_{T}\|v\|_{H^{1}\left(S_{T}\right)}+h_{T}^{3 / 2}\left\|g^{\prime}\right\|_{L^{2}\left(\partial S_{T} \cap \partial \Omega\right)}\right) \\
\left\|v-\pi_{h} v\right\|_{L^{2}(T)} & \leq C h_{T}^{2}\|v\|_{H^{2}\left(S_{T}\right)} \quad \text { if } v \in H^{2}(T), \\
\left\|\nabla\left(v-\pi_{h} v\right)\right\|_{L^{2}(T)} & \leq C\left(\|\nabla v\|_{L^{2}\left(S_{T}\right)}+h_{T}^{1 / 2}\left\|g^{\prime}\right\|_{L^{2}\left(\partial S_{T} \cap \partial \Omega\right)}\right) \quad \text { if } v \in H^{1}(T), \\
\left\|\nabla\left(v-\pi_{h} v\right)\right\|_{L^{2}(T)} & \leq C h_{T}\|v\|_{H^{2}\left(S_{T}\right)} \quad \text { if } v \in H^{2}(T) .
\end{aligned}
$$

Proof. In the case if $T \in \mathcal{T}_{h}$ with $T \cap \partial \Omega=\emptyset$, then the estimates are direct consequence of the stability and polynomial invariance of the interpolation operator $\pi_{h}$. Now let $T \in \mathcal{T}_{h}$ with $T \cap \partial \Omega \neq \emptyset$. Then by using (3.7), we find for any $p \in \mathbb{P}_{1}(T)$ that

$$
\begin{aligned}
\left\|v-\pi_{h} v\right\|_{L^{2}(T)} & =\left\|v+p-\pi_{h}(v+p)\right\|_{L^{2}(T)} \leq\|v+p\|_{L^{2}(T)}+\left\|\pi_{h}(v+p)\right\|_{L^{2}(T)} \\
& \leq C\left(\|v+p\|_{L^{2}\left(S_{T}\right)}+h_{T}\|\nabla(v+p)\|_{L^{2}\left(S_{T}\right)}+h_{T}^{3 / 2}\left\|(g+p)^{\prime}\right\|_{L^{2}\left(\partial S_{T} \cap \partial \Omega\right)}\right) .
\end{aligned}
$$

If $v \in H^{1}(T)$, then choose $p \in \mathbb{P}_{0}(T)$ as the integral mean of $v$ on $S_{T}$. If $v \in H^{2}(T)$, then choose $p \in \mathbb{P}_{1}(T)$ as the Lagrange interpolation of $v$ and complete the proof of the $L^{2}$-norm estimate. Using similar arguments we can derive the estimates for the derivative. For

$$
\begin{aligned}
\left\|\nabla\left(v-\pi_{h} v\right)\right\|_{L^{2}(T)} & =\left\|\nabla\left(v+p-\pi_{h}(v+p)\right)\right\|_{L^{2}(T)} \leq\|\nabla(v+p)\|_{L^{2}(T)}+\left\|\nabla \pi_{h}(v+p)\right\|_{L^{2}(T)} \\
& \leq C\left(\|\nabla(v+p)\|_{L^{2}\left(S_{T}\right)}+h_{T}^{1 / 2}\left\|(g+p)^{\prime}\right\|_{L^{2}\left(\partial S_{T} \cap \partial \Omega\right)}\right) .
\end{aligned}
$$

Choosing appropriate $p$ completes the proof.

\subsection{Discrete solution with sharp boundary condition}

The numerical method in (3.1)-(3.2) can be treated as a nonconforming scheme with respect to the Dirichlet data since the discrete problem uses the approximate Dirichlet data $-\psi_{h}$ which is not equal to the given data $-\psi$ on the boundary $\partial \Omega$, where $\psi_{h}$ is the linear Lagrange interpolation of $\psi$. Following the details in [30], we construct an intermediate solution $\tilde{W}_{h}^{n}$ (which can be treated as a post-processing of $W_{h}^{n}$ ) with the help of $W_{h}^{n}$ and $\psi$ as follows. For $T \in \mathcal{T}_{h}^{i}$, define $\tilde{W}_{h}^{n}$ to be the same as $W_{h}^{n}$ on $T$. For $T \in \mathcal{T}_{h}^{b}$, first define $W_{h}^{n *}$ on $\partial T$ (the boundary of $T$ ) by

$$
W_{h}^{n *}(x, y):= \begin{cases}-\psi(x, y) & \text { if }(x, y) \in \partial \Omega, \\ W_{h}^{n}(x, y) & \text { if }(x, y) \in \partial T \backslash \partial \Omega .\end{cases}
$$




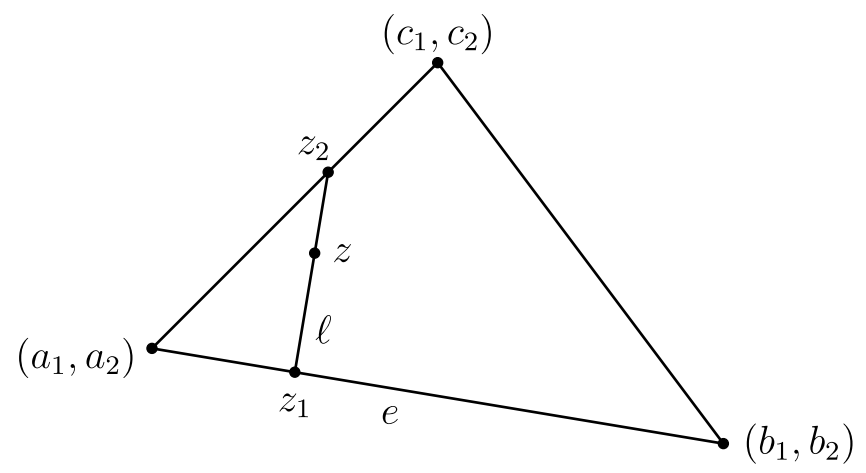

Fig. 3.1. Heights $h_{1}$ and $h_{2}$.

Then define $\tilde{W}_{h}^{n}$ to be some $H^{1}(T)$ extension of $W_{h}^{n *} \in C(\partial T)$ to $T$ such that $\left.\tilde{W}_{h}^{n}\right|_{\partial T}=W_{h}^{n *}$ and $\left.\tilde{W}_{h}^{n}\right|_{T}=\left.W_{h}^{n}\right|_{T}$ for all $T \in \mathcal{T}_{h}^{i}$. By the construction $\tilde{W}_{h}^{n} \in H^{1}(\Omega)$ and $W_{h}^{n}-\tilde{W}_{h}^{n} \equiv 0$ on any triangle $T \in \mathcal{T}_{h}^{i}$.

Let $T \in \mathcal{T}_{h}^{b}$ and $W_{h}^{n *} \in C(\partial T)$ be defined by (3.16). Let $e \in \mathcal{E}_{h}^{b}$ be such that $e \subset \partial T$. Let $z=(x, y)$ be an interior point of $T$ and $\ell$ be the line that is orthogonal to $e$ passing through $z$, as it shown in Fig. 3.1. Let $z_{1}=\left(x_{1}, y_{1}\right)$ and $z_{2}=\left(x_{2}, y_{2}\right)$ be the points of intersection of $\ell$ with $\partial T$. Let $h_{1}$ is the distance between $z$ and $z_{1}$; and $h_{2}$ is distance between $z$ and $z_{2}$. Then define $\tilde{W}_{h}^{n}$ on $T$ by

$$
\tilde{W}_{h}^{n}(x, y):=\frac{h_{2} W_{h}^{n *}\left(x_{1}, y_{1}\right)+h_{1} W_{h}^{n *}\left(x_{2}, y_{2}\right)}{h_{1}+h_{2}} \quad \text { for }(x, y) \in T^{\circ},
$$

where $T^{\circ}$ is the interior of $T$.

Let $T \in \mathcal{T}_{h}^{b}$. Since $\left.W_{h}^{n}\right|_{T} \in \mathbb{P}_{1}(T)$, we note that

$$
W_{h}^{n}(x, y)=\frac{h_{2} W_{h}^{n}\left(x_{1}, y_{1}\right)+h_{1} W_{h}^{n}\left(x_{2}, y_{2}\right)}{h_{1}+h_{2}} \quad \text { for }(x, y) \in T^{\circ},
$$

and

$$
\left(\tilde{W}_{h}^{n}-W_{h}^{n}\right)(x, y)=\left(\frac{h_{2}}{h_{1}+h_{2}}\right)\left(\psi_{h}-\psi\right)\left(x_{1}, y_{1}\right) .
$$

Since $\left|h_{2} /\left(h_{1}+h_{2}\right)\right| \leq 1$, the following result is immediate, see [30].

Theorem 3.3. Let $T \in \mathcal{T}_{h}^{b}$ and define $\tilde{W}_{h}^{n}$ by (3.17), a linear extension of $W_{h}^{n *}$ given by (3.16). Let $e \in \mathcal{E}_{h}^{b}$ be an edge of $T$ and assume $\psi$ is such that $\left.\psi\right|_{e} \in H^{1}(e)$. Then there holds

$$
\left\|\tilde{W}_{h}^{n}-W_{h}^{n}\right\|_{L^{2}(T)} \leq C h_{e}^{\frac{1}{2}}\left\|\psi-\psi_{h}\right\|_{L^{2}(e)},
$$

where $C$ is a positive constant, $e \in \mathcal{E}_{h}^{b}$ an edge of $T$. Moreover,

$$
\left\|\tilde{W}_{h}^{n}-W_{h}^{n}\right\|_{L^{2}(\Omega)} \leq C\left(\sum_{e \in \mathcal{E}_{h}^{b}} h_{e}\left\|\psi-\psi_{h}\right\|_{L^{2}(e)}^{2}\right)^{1 / 2} .
$$

From Theorem 3.3 and [30, Theorem 3.2], we have the following corollary. The corollary establishes the difference between the numerical solution $W_{h}^{n}$ of variational inequality (3.1)-(3.2) and post-processing of $W_{h}^{n}$ in the $L^{2}$ and the $H^{1}$ norms. This corollary plays a crucial rule in the subsequent a priori error analysis to obtain optimal order of convergence.

Corollary 3.4. For each $W_{h}^{n}$ there exists $\tilde{W}_{h}^{n} \in \mathcal{K}$ such that

$$
\left\|\tilde{W}_{h}^{n}-W_{h}^{n}\right\|_{L^{2}(\Omega)} \leq C h^{2} \text { and }\left\|\tilde{W}_{h}^{n}-W_{h}^{n}\right\|_{H^{1}(\Omega)} \leq C h .
$$

\section{Error estimate}

In this section we estimate the error i.e., the difference between the solution of parabolic variational inequality (2.4)-(2.5) and its numerical approximation as described in Section 3. We show that this error estimated in a certain norm, is of order $h+(\Delta t)^{\frac{3}{4}}\left(\log \frac{1}{\Delta t}\right)^{\frac{1}{4}}$. 
Theorem 4.1 (Main Theorem). Suppose $w$ is the solution of (2.4)-(2.5) and,

$$
f \in C\left(\mathcal{J} ; L^{\infty}(\Omega)\right), \quad \frac{\partial f}{\partial t} \in L^{2}\left(\mathcal{J} ; L^{\infty}(\Omega)\right), \quad w_{0} \in W^{2, \infty}(\Omega) \cap \mathcal{K},
$$

and (3.3) holds. Let $W_{h}$ be the solution of the corresponding discrete problem (3.1). Then, there exists a constant $C$ independent of $\Delta t$ and $h$ such that

$$
\max _{n \in\{1, \ldots, N\}}\left\|w^{n}-W_{h}^{n}\right\|_{L^{2}(\Omega)}+\left(\sum_{n=1}^{N}\left\|w^{n}-W_{h}^{n}\right\|_{H^{1}(\Omega)}^{2} \Delta t\right)^{\frac{1}{2}} \leq C\left[h+(\Delta t)^{\frac{3}{4}}\left(\log \frac{1}{\Delta t}\right)^{\frac{1}{4}}\right],
$$

where $w^{n}$ and $W_{h}^{n}$ is the solution at time $t_{n}$.

Proof. Let us define $\eta(t):=w(t)-\pi_{h} w(t)$ for $t \in \mathcal{J}, e^{n}:=w^{n}-W_{h}^{n}$ and $\tilde{e}^{n}:=w^{n}-\tilde{W}_{h}^{n}$ for $n=0, \ldots, N$. Then, $e^{n}-\eta^{n}=\pi_{h} w^{n}-W_{h}^{n}$. Now,

$$
\begin{aligned}
\left(\partial e^{n}, e^{n}\right)+a\left(e^{n}, e^{n}\right)= & \left(\partial e^{n}, \eta^{n}\right)+\left(\partial e^{n}, e^{n}-\eta^{n}\right)+a\left(e^{n}, \eta^{n}\right) \\
& +a\left(e^{n}, e^{n}-\eta^{n}\right) \\
= & \left(\partial e^{n}, \eta^{n}\right)+a\left(e^{n}, \eta^{n}\right)+\left(\partial w^{n}, \pi_{h} w^{n}-W_{h}^{n}\right) \\
& -\left(\partial W_{h}^{n}, \pi_{h} w^{n}-W_{h}^{n}\right)+a\left(w^{n}-W_{h}^{n}, \pi_{h} w^{n}-W_{h}^{n}\right) .
\end{aligned}
$$

First we put $v=\tilde{W}_{h}^{n} \in \mathcal{K}$ and $t=t_{n}$ in the inequality of (2.6) and $v=\pi_{h} w^{n} \in \mathcal{K}_{h}$ in (3.1), then by adding these two inequalities, we get

$$
\begin{aligned}
-\left(\partial W_{h}^{n}, \pi_{h} w^{n}-W_{h}^{n}\right) & -a\left(W_{h}^{n}, \pi_{h} w^{n}-W_{h}^{n}\right) \\
\leq & \left(f^{n}+\Delta \psi, \eta^{n}\right)-\left(f^{n}+\Delta \psi, \tilde{W}_{h}^{n}-W_{h}^{n}\right) \\
& +\left(\frac{\partial^{+} w}{\partial t}\left(t_{n}\right), \tilde{W}_{h}^{n}-w^{n}\right)+a\left(w^{n}, \tilde{W}_{h}^{n}-w^{n}\right) .
\end{aligned}
$$

By applying inequality (4.2) in Eq. (4.1) it follows that

$$
\begin{aligned}
\left(\partial e^{n}, e^{n}\right)+a\left(e^{n}, e^{n}\right) \leq \sum_{j=1}^{6} A_{n}^{j}, \\
\text { where } A_{n}^{1}=\left(\partial e^{n}, \eta^{n}\right) \\
A_{n}^{2}=a\left(e^{n}, \eta^{n}\right) \\
A_{n}^{3}=\left(f^{n}+\Delta \psi, \eta^{n}\right)-a\left(w^{n}, \eta^{n}\right)+a\left(w^{n}, \tilde{W}_{h}^{n}-W_{h}^{n}\right) \\
-\left(f^{n}+\Delta \psi, \tilde{W}_{h}^{n}-W_{h}^{n}\right) \\
A_{n}^{4}=\left(\frac{\partial^{+} w}{\partial t}\left(t_{n}\right)-\partial w^{n}, W_{h}^{n}-w^{n}\right) \\
A_{n}^{5}=\left(\frac{\partial^{+} w}{\partial t}\left(t_{n}\right), \tilde{W}_{h}^{n}-W_{h}^{n}\right) \\
A_{n}^{6}=-\left(\partial w^{n}, \eta^{n}\right) .
\end{aligned}
$$

By multiplying (4.3) with $\Delta t$ and taking summation on both sides over $n=1, \ldots, N$, we get

$$
\begin{array}{r}
\sum_{n=1}^{N}\left(e^{n}-e^{n-1}, e^{n}\right)+\sum_{n=1}^{N} a\left(e^{n}, e^{n}\right) \Delta t \leq \sum_{n=1}^{N} \sum_{j=1}^{6} A_{n}^{j} \Delta t=\sum_{j=1}^{6} E_{j}, \\
\text { where } E_{j}=\sum_{n=1}^{N} A_{n}^{j} \Delta t, \text { for } j=1,2, \ldots, 6 .
\end{array}
$$

Since

$$
\begin{aligned}
2 \sum_{n=1}^{M}\left(e^{n}-e^{n-1}, e^{n}\right) & =\sum_{n=1}^{M}\left(e^{n}-e^{n-1}, e^{n}-e^{n-1}\right)+\sum_{n=1}^{M}\left(e^{n}-e^{n-1}, e^{n-1}\right)+\sum_{n=1}^{M}\left(e^{n}-e^{n-1}, e^{n}\right) \\
& =\sum_{n=1}^{M}\left\|e^{n}-e^{n-1}\right\|_{L^{2}(\Omega)}^{2}+\left\|e^{M}\right\|_{L^{2}(\Omega)}^{2}-\left\|e^{0}\right\|_{L^{2}(\Omega)}^{2} \text { for } M=1, \ldots, N,
\end{aligned}
$$


inequality (4.4) yields

$$
\max _{n \in\{1, \ldots, N\}}\left\|e^{n}\right\|_{L^{2}(\Omega)}^{2}+2 \sum_{n=1}^{N} a\left(e^{n}, e^{n}\right) \Delta t \leq\left\|e^{0}\right\|_{L^{2}(\Omega)}^{2}+2 \sum_{j=1}^{6} E_{j} .
$$

We estimate the terms $E_{i}, i=1, \ldots, 6$ in the subsequent discussion.

Estimation of $E_{1}$ :

$$
\begin{aligned}
E_{1}=\sum_{n=1}^{N}\left(\partial e^{n}, \eta^{n}\right) \Delta t & =\sum_{n=1}^{N}\left(e^{n}, \eta^{n}\right)-\sum_{n=1}^{N}\left(e^{n-1}, \eta^{n}\right) \\
& =\sum_{n=1}^{N}\left(e^{n-1}, \eta^{n-1}\right)+\left(e^{N}, \eta^{N}\right)-\left(e^{0}, \eta^{0}\right)-\sum_{n=1}^{N}\left(e^{n-1}, \eta^{n}\right) \\
& =-\sum_{n=1}^{N}\left(e^{n-1}, \partial \eta^{n}\right) \Delta t+\left(e^{N}, \eta^{N}\right)-\left(e^{0}, \eta^{0}\right) .
\end{aligned}
$$

By using Hölder's inequality and Cauchy-Schwarz inequality, we deduce

$$
\begin{aligned}
E_{1} \leq & \sum_{n=1}^{N}\left\|e^{n-1}\right\|_{L^{2}(\Omega)}\left\|\partial \eta^{n}\right\|_{L^{2}(\Omega)} \Delta t+\left\|e^{N}\right\|_{L^{2}(\Omega)}\left\|\eta^{N}\right\|_{L^{2}(\Omega)}+\left\|e^{0}\right\|_{L^{2}(\Omega)}\left\|\eta^{0}\right\|_{L^{2}(\Omega)} \\
\leq & \frac{\epsilon}{2} \sum_{n=1}^{N}\left\|e^{n-1}\right\|_{L^{2}(\Omega)}^{2} \Delta t+\frac{1}{2 \epsilon} \sum_{n=0}^{N-1}\left\|\partial \eta^{n}\right\|_{L^{2}(\Omega)}^{2} \Delta t+\frac{\epsilon}{2}\left\|e^{N}\right\|_{L^{2}(\Omega)}^{2} \\
& +\frac{1}{2 \epsilon}\left\|\eta^{N}\right\|_{L^{2}(\Omega)}^{2}+\frac{\epsilon}{2}\left\|e^{0}\right\|_{L^{2}(\Omega)}^{2}+\frac{1}{2 \epsilon}\left\|\eta^{0}\right\|_{L^{2}(\Omega)}^{2} .
\end{aligned}
$$

From (2.6) we have $w^{n} \in H^{2}(\Omega)$, hence from (3.10) and (3.13) of Theorem 3.2, we get

$$
\begin{aligned}
\left\|\eta^{n}\right\|_{L^{2}(\Omega)} & \leq C h^{2}\left(\sum_{T \in T_{h}}\left\|w^{n}\right\|_{H^{2}\left(S_{T}\right)}^{2}\right)^{\frac{1}{2}} \\
& \leq C h^{2} \max _{n}\left\|w^{n}\right\|_{H^{2}(\Omega)} \\
& \leq C h^{2}\|w\|_{L^{\infty}\left(\mathcal{J} ; H^{2}(\Omega)\right)} .
\end{aligned}
$$

Therefore, $\max _{n}\left\|\eta^{n}\right\|_{L^{2}(\Omega)} \leq C h^{2}\|w\|_{L^{\infty}\left(\mathcal{J} ; H^{2}(\Omega)\right)}$. On the other hand

$$
\left\|\partial \eta^{n}\right\|_{L^{2}(\Omega)} \leq(\Delta t)^{-\frac{1}{2}}\left\|\frac{\partial \eta}{\partial t}\right\|_{L^{2}\left(\mathcal{J}_{n} ; L^{2}(\Omega)\right)}
$$

Since time differentiation commutes with interpolation $\pi_{h}$ and $\frac{\partial w}{\partial t} \in L^{2}\left(\mathcal{J} ; H_{0}^{1}(\Omega)\right.$ ), by (3.10) and (3.12) of Theorem 3.2, we have

$$
\left\|\frac{\partial \eta(t)}{\partial t}\right\|_{L^{2}(\Omega)} \leq C h\left\|\frac{\partial w(t)}{\partial t}\right\|_{H^{1}(\Omega)} .
$$

This implies,

$$
\left\|\frac{\partial \eta}{\partial t}\right\|_{L^{2}\left(\mathcal{J}_{n} ; L^{2}(\Omega)\right)} \leq C h\left\|\frac{\partial w}{\partial t}\right\|_{L^{2}\left(\mathcal{J}_{n} ; H^{1}(\Omega)\right)} .
$$

Therefore, $\left\|\partial \eta^{n}\right\|_{L^{2}(\Omega)} \leq C h(\Delta t)^{-\frac{1}{2}}\left\|\frac{\partial w}{\partial t}\right\|_{L^{2}\left(\mathcal{J}_{n} ; H^{1}(\Omega)\right)}$.

By using above inequalities in (4.6), we get the following.

$$
\begin{aligned}
E_{1} \leq & \frac{T \epsilon}{2} \max _{n \in\{1, \ldots, N\}}\left\|e^{n-1}\right\|_{L^{2}(\Omega)}^{2}+C h^{2} \sum_{n=1}^{N}(\Delta t)^{-1}\left\|\frac{\partial w}{\partial t}\right\|_{L^{2}\left(\mathcal{J}_{n} ; H^{1}(\Omega)\right)}^{2} \Delta t+\frac{\epsilon}{2}\left\|e^{N}\right\|_{L^{2}(\Omega)}^{2} \\
& +C h^{4}\|w\|_{L^{\infty}\left(\mathcal{J} ; H^{2}(\Omega)\right)}^{2}+\frac{\epsilon}{2}\left\|e^{0}\right\|_{L^{2}(\Omega)}^{2} \\
\leq & \frac{T \epsilon}{2} \max _{n \in\{1, \ldots, N\}}\left\|e^{n-1}\right\|_{L^{2}(\Omega)}^{2}+C h^{2}\left\|\frac{\partial w}{\partial t}\right\|_{L^{2}\left(\mathcal{J} ; H^{1}(\Omega)\right)}^{2}+\frac{\epsilon}{2}\left\|e^{N}\right\|_{L^{2}(\Omega)}^{2}+\frac{\epsilon}{2}\left\|e^{0}\right\|_{L^{2}(\Omega)}^{2} \\
& \left.+C h^{4}\|w\|_{L^{\infty}\left(\mathcal{J} ; H^{2}(\Omega)\right)}^{2} \text { (by using } N=\frac{T}{\Delta t}\right) .
\end{aligned}
$$


Estimation of $E_{2}$ :

$E_{2}=\sum_{n=1}^{N} A_{n}^{2} \Delta t$. By using Hölder's inequality and Cauchy-Schwarz inequality, we get

$$
\left|A_{n}^{2}\right| \Delta t=\left|a\left(e^{n}, \eta^{n}\right)\right| \Delta t \leq \frac{\epsilon \Delta t}{2}\left\|e^{n}\right\|_{H^{1}(\Omega)}^{2}+\frac{1}{2 \epsilon}\left\|\eta^{n}\right\|_{H^{1}(\Omega)}^{2} \Delta t .
$$

By using (3.11) and (3.15) of Theorem 3.2,

$$
\begin{aligned}
\left\|\eta^{n}\right\|_{H^{1}(\Omega)}^{2} & \leq C \sum_{T \in \mathcal{T}_{h}} h_{T}^{2}\left\|w^{n}\right\|_{H^{2}\left(S_{T}\right)}^{2} \\
& \leq C h^{2}\left\|w^{n}\right\|_{H^{2}(\Omega)}^{2} \\
& \leq C h^{2}\|w\|_{L^{\infty}\left(\mathcal{J} ; H^{2}(\Omega)\right)}^{2} .
\end{aligned}
$$

From the above inequalities in Estimation of $E_{2}$ and the fact $N=\frac{T}{\Delta t}$, we have

$$
E_{2} \leq \frac{T \epsilon}{2} \max _{n \in\{1, \ldots, N\}}\left\|e^{n}\right\|_{L(\Omega)}^{2}+\frac{\epsilon}{2} \sum_{n=1}^{N} a\left(e^{n}, e^{n}\right) \Delta t+C h^{2}\|w\|_{L^{\infty}\left(\mathcal{J} ; H^{2}(\Omega)\right)}^{2} .
$$

\section{Estimation of $E_{3}$ :}

$E_{3}=\sum_{n=1}^{N} A_{n}^{3} \Delta t$, where

$$
A_{n}^{3}=\left(f^{n}+\Delta \psi, \eta^{n}\right)-\left(f^{n}+\Delta \psi, \tilde{W}_{h}^{n}-W_{h}^{n}\right)-a\left(w^{n}, \eta^{n}-\left(\tilde{W}_{h}^{n}-W_{h}^{n}\right)\right) .
$$

By applying integration by parts in the last term of right hand side, we have

$$
A_{n}^{3}=\left(f^{n}+\Delta \psi, \eta^{n}\right)-\left(f^{n}+\Delta \psi, \tilde{W}_{h}^{n}-W_{h}^{n}\right)+\int_{\Omega} \Delta w^{n}\left(\eta^{n}-\left(\tilde{W}_{h}^{n}-W_{h}^{n}\right)\right) d x,
$$

where we have used the fact that $\eta^{n}-\left(\tilde{W}_{h}^{n}-W_{h}^{n}\right) \in H_{0}^{1}(\Omega)$. Then by applying Hölder's inequality and triangle inequality, we get

$$
\left|A_{n}^{3}\right| \leq\left(\left\|f^{n}\right\|_{L^{2}(\Omega)}+\|\Delta \psi\|_{L^{2}(\Omega)}+\left\|\Delta w^{n}\right\|_{L^{2}(\Omega)}\right)\left(\left\|\eta^{n}\right\|_{L^{2}(\Omega)}+\left\|\tilde{W}_{h}^{n}-W_{h}^{n}\right\|_{L^{2}(\Omega)}\right) .
$$

Thus,

$$
\begin{aligned}
E_{3} \leq & \left(\|f\|_{L^{\infty}\left(\mathcal{J} ; L^{2}(\Omega)\right)}+\|\Delta \psi\|_{L^{2}(\Omega)}+\|\Delta w\|_{L^{\infty}\left(\mathcal{J} ; L^{2}(\Omega)\right)}\right) \sum_{n=1}^{N} \Delta t\left(\left\|\eta^{n}\right\|_{L^{2}(\Omega)}\right. \\
& \left.+\left\|\tilde{W}_{h}^{n}-W_{h}^{n}\right\|_{L^{2}(\Omega)}\right) .
\end{aligned}
$$

Now by using Theorem 3.2 and Corollary 3.4, we have

$$
\begin{aligned}
E_{3} & \leq C h^{2}\left(\|f\|_{L^{\infty}\left(\mathcal{J} ; L^{2}(\Omega)\right)}+\|\Delta \psi\|_{L^{2}(\Omega)}+\|\Delta w\|_{L^{\infty}\left(\mathcal{J} ; L^{2}(\Omega)\right)}\right)\left(\sum_{n=1}^{N} \Delta t\left\|w^{n}\right\|_{H^{2}(\Omega)}+\sum_{n=1}^{N} \Delta t\right) \\
& \leq C h^{2}\left(\|f\|_{L^{\infty}\left(\mathcal{J} ; L^{2}(\Omega)\right)}+\|\Delta \psi\|_{L^{2}(\Omega)}+\|\Delta w\|_{L^{\infty}\left(\mathcal{J} ; L^{2}(\Omega)\right)}\right)\left(N \Delta t\|w\|_{L^{\infty}\left(\mathcal{J} ; L^{2}(\Omega)\right)}+N \Delta t\right) .
\end{aligned}
$$

From the fact $f \in C\left(\mathcal{J} ; L^{\infty}(\Omega)\right), w \in L^{\infty}\left(\mathcal{J} ; W^{2, p}(\Omega)\right), 1 \leq p<\infty$ and $\psi \in H^{2}(\Omega)$ with $N=\frac{T}{\Delta t}$ we deduce

$$
E_{3} \leq C h^{2} \text {. }
$$

\section{Estimation of $E_{4}$ :}

$$
E_{4}=\sum_{n=1}^{N} A_{n}^{4} \Delta t \text {, where }
$$

$$
\begin{aligned}
A_{n}^{4}= & \left(\partial w^{n}-\frac{\partial^{+} w}{\partial t}\left(t_{n}\right), w^{n}-W_{h}^{n}\right) \\
= & \int_{\Omega}\left(\frac{w^{n}-w^{n-1}}{\Delta t}-\frac{\partial^{+} w}{\partial t}\left(t_{n}\right)\right) e^{n} d x \\
= & \frac{1}{\Delta t} \int_{\mathcal{J}_{n}}\left[\int_{\Omega}\left(\frac{\partial^{+} w}{\partial t}(t)-\frac{\partial^{+} w}{\partial t}\left(t_{n}\right)\right) e^{n} d x\right] d t \\
= & \frac{1}{\Delta t} \int_{\mathcal{J}_{n}}\left[\int_{\Omega^{+}(t)} \Delta w(t) e^{n} d x-\int_{\Omega^{+}\left(t_{n}\right)} \Delta w\left(t_{n}\right) e^{n} d x\right] d t \quad(\text { by }(2.7)) \\
& +\frac{1}{\Delta t} \int_{\mathcal{J}_{n}}\left[\int_{\Omega^{+}(t)}(f(t)+\Delta \psi) e^{n} d x-\int_{\Omega^{+}\left(t_{n}\right)}\left(f^{n}+\Delta \psi\right) e^{n} d x\right] d t
\end{aligned}
$$




$$
\begin{aligned}
+ & \frac{1}{\Delta t} \int_{\mathcal{J}_{n}} \int_{\Omega^{0}(t)} \max \{f(x, t)+\Delta \psi(x), 0\} e^{n} d x d t \\
& -\frac{1}{\Delta t} \int_{\mathcal{J}_{n}} \int_{\Omega^{0}\left(t_{n}\right)} \max \left\{f\left(x, t_{n}\right)+\Delta \psi(x), 0\right\} e^{n} d x d t \\
= & \frac{1}{\Delta t} \int_{\mathcal{J}_{n}}\left[\int_{\Omega^{+}(t)} \Delta w(t) e^{n} d x-\int_{\Omega^{+}\left(t_{n}\right)} \Delta w\left(t_{n}\right) e^{n} d x\right] d t \\
& +\frac{1}{\Delta t} \int_{\mathcal{J}_{n}}\left[\int_{\Omega}\left(\tilde{f}(x, t)-\tilde{f}\left(x, t_{n}\right)\right) e^{n} d x\right] d t \\
= & B_{n}^{1}+B_{n}^{2},
\end{aligned}
$$

here $\tilde{f}(x, t)=\left\{\begin{array}{lll}f(x, t)+\Delta \psi(x) & \text { if } & x \in \Omega^{+}(t) \\ \max \{0, f(x, t)+\Delta \psi(x)\} & \text { if } & x \in \Omega^{0}(t) .\end{array}\right.$.

Now, we use the fact $\Delta w(t)=0$ a.e. on $\Omega^{0}(t)$ to estimate $B_{n}^{1}$.

$$
\begin{aligned}
B_{n}^{1}= & \frac{1}{\Delta t} \int_{\mathcal{J}_{n}}\left[\int_{\Omega^{+}(t)} \Delta w(t) e^{n} d x-\int_{\Omega^{+}\left(t_{n}\right)} \Delta w\left(t_{n}\right) e^{n} d x\right] d t \\
= & \frac{1}{\Delta t} \int_{\mathcal{J}_{n}} \int_{\Omega}\left[\Delta w(t)-\Delta w\left(t_{n}\right)\right] e^{n} d x d t \\
= & \frac{1}{\Delta t} \int_{\mathcal{J}_{n}} \int_{\Omega}\left[\Delta w(t)-\Delta w\left(t_{n}\right)\right] \tilde{e}^{n} d x d t \\
& +\frac{1}{\Delta t} \int_{\mathcal{J}_{n}} \int_{\Omega}\left[\Delta w(t)-\Delta w\left(t_{n}\right)\right]\left(e^{n}-\tilde{e}^{n}\right) d x d t \\
& =I_{1}+I_{2} .
\end{aligned}
$$

By using integration by parts over $\Omega$, we have the following.

$$
\begin{aligned}
I_{1} & =\frac{1}{\Delta t} \int_{\mathcal{J}_{n}} \int_{\Omega}\left[\Delta w(t)-\Delta w\left(t_{n}\right)\right] \tilde{e}^{n} d x d t \\
& =\frac{1}{\Delta t} \int_{\mathcal{J}_{n}} \int_{\Omega}\left(\nabla w\left(t_{n}\right)-\nabla w(t)\right) \cdot \nabla \tilde{e}^{n} d x d t \\
& =\frac{1}{\Delta t} \int_{\mathcal{J}_{n}} \int_{t}^{t_{n}} a\left(\frac{\partial w}{\partial t}(s), \tilde{e}^{n}\right) d s d t .
\end{aligned}
$$

By using Hölder's inequality, Cauchy-Schwarz inequality and triangle inequality we deduce

$$
\begin{aligned}
\left|I_{1}\right| & \leq \frac{1}{\Delta t} \int_{\mathcal{J}_{n}} \int_{\mathcal{J}_{n}}\left\|\tilde{e}^{n}\right\|_{H^{1}(\Omega)}\left\|\frac{\partial w}{\partial t}(s)\right\|_{H^{1}(\Omega)} d s d t \\
& \leq \frac{1}{\Delta t}\left\|\tilde{e}^{n}\right\|_{H^{1}(\Omega)} \int_{\mathcal{J}_{n}}\left[\left(\int_{\mathcal{J}_{n}}\left\|\frac{\partial w}{\partial t}(s)\right\|_{H^{1}(\Omega)}^{2}\right)^{\frac{1}{2}}(\Delta t)^{\frac{1}{2}}\right] d t \\
& =(\Delta t)^{\frac{1}{2}}\left\|\tilde{e}^{n}\right\|_{H^{1}(\Omega)}\left\|\frac{\partial w}{\partial t}\right\|_{L^{2}\left(\mathcal{J}_{n} ; H^{1}(\Omega)\right)} \\
& \leq \frac{\epsilon}{2}\left\|\tilde{e}^{n}\right\|_{H^{1}(\Omega)}^{2}+\frac{1}{2 \epsilon} \Delta t\left\|\frac{\partial w}{\partial t}\right\|_{L^{2}\left(\mathcal{J}_{n} ; H^{1}(\Omega)\right)}^{2} \\
& \leq \frac{\epsilon}{2}\left\|e^{n}\right\|_{H^{1}(\Omega)}^{2}+\frac{\epsilon}{2}\left\|W_{h}^{n}-\tilde{W}_{h}^{n}\right\|_{H^{1}(\Omega)}^{2}+\frac{1}{2 \epsilon} \Delta t\left\|\frac{\partial w}{\partial t}\right\|_{L^{2}\left(\mathcal{J}_{n} ; H^{1}(\Omega)\right)}^{2}
\end{aligned}
$$

On the other hand $I_{2}=\frac{1}{\Delta t} \int_{\mathcal{J}_{n}} \int_{\Omega}\left(\Delta w(t)-\Delta w\left(t_{n}\right)\right)\left(e^{n}-\tilde{e}^{n}\right) d x d t$.

By using Hölder's inequality, triangle inequality and the fact $w \in L^{\infty}\left(\mathcal{J} ; W^{2, p}(\Omega)\right), \quad 1 \leq p<\infty$ we deduce

$$
\begin{aligned}
\left|I_{2}\right| & \leq \frac{1}{\Delta t} \int_{\mathcal{J}_{n}}\left(\|\Delta w(t)\|_{L^{2}(\Omega)}+\left\|\Delta w\left(t_{n}\right)\right\|_{L^{2}(\Omega)}\right)\left\|e^{n}-\tilde{e}^{n}\right\|_{L^{2}(\Omega)} d t \\
& \leq 2\|\Delta w\|_{L^{\infty}\left(\mathcal{J} ; L^{2}(\Omega)\right)}\left\|e^{n}-\tilde{e}^{n}\right\|_{L^{2}(\Omega)} .
\end{aligned}
$$


Since $e^{n}-\tilde{e^{n}}=W_{h}^{n}-\tilde{W}_{h}^{n}$ and $\Delta t=\frac{T}{N}$, we have

$$
\begin{aligned}
\sum_{n=1}^{N}\left|B_{n}^{1}\right| \Delta t \leq & \sum_{n=1}^{N}\left|I_{1}\right| \Delta t+\sum_{n=1}^{N}\left|I_{2}\right| \Delta t \\
\leq & \frac{\epsilon}{2} \sum_{n=1}^{N}\left\|e^{n}\right\|_{H^{1}(\Omega)}^{2} \Delta t+\frac{T \epsilon}{2} \max _{n \in\{1, \ldots, N\}}\left\|W_{h}^{n}-\tilde{W}_{h}^{n}\right\|_{H^{1}(\Omega)}^{2} \\
& +\frac{1}{2 \epsilon}(\Delta t)^{2}\left\|\frac{\partial w}{\partial t}\right\|_{L^{2}\left(\mathcal{J} ; H^{1}(\Omega)\right)}^{2} \\
& +2 T\|\Delta w\|_{L^{\infty}\left(\mathcal{J} ; L^{2}(\Omega)\right)} \max _{n \in\{1, \ldots, N\}}\left\|W_{h}^{n}-\tilde{W}_{h}^{n}\right\|_{L^{2}(\Omega)} .
\end{aligned}
$$

Now we estimate $B_{n}^{2}$. The estimation of $B_{n}^{2}$ is similar to the estimation of $q_{n}^{2}$ in [1, page no. 605]. But for the sake of the completeness we have given the following details:

$$
\begin{aligned}
B_{n}^{2}= & \frac{1}{\Delta t} \int_{\mathcal{J}_{n}}\left[\int_{\Omega}\left(\tilde{f}(x, t)-\tilde{f}\left(x, t_{n}\right)\right) e^{n} d x\right] d t \\
= & \frac{1}{\Delta t} \int_{\mathcal{J}_{n}}\left[\int_{\Omega \backslash D_{n}}\left(\tilde{f}(x, t)-\tilde{f}\left(x, t_{n}\right)\right) e^{n} d x\right] d t \\
& +\frac{1}{\Delta t} \int_{\mathcal{J}_{n}}\left[\int_{D_{n}}\left(\tilde{f}(x, t)-\tilde{f}\left(x, t_{n}\right)\right) e^{n} d x\right] d t .
\end{aligned}
$$

Now, we prove the following inequality

$$
\left|\tilde{f}(x, t)-\tilde{f}\left(x, t_{n}\right)\right| \leq\left|f(x, t)-f\left(x, t_{n}\right)\right| \text { for } \Omega \backslash D_{n}, t \in \mathcal{J}_{n} .
$$

Let us choose a point $x \in \Omega \backslash D_{n}$.

Case 1. If $x \in \Omega^{+}\left(t_{n}\right)$ then, $x \in \Omega^{+}(t)$ from the definition of $D_{n}$ in (3.3). Therefore, $\left|\tilde{f}(x, t)-\tilde{f}\left(x, t_{n}\right)\right|=$ $\left|f(x, t)+\Delta \psi(x)-f\left(x, t_{n}\right)-\Delta \psi(x)\right|$.

Case 2. If $x \notin \Omega^{+}\left(t_{n}\right)$ then, $x \in \cup_{t \in \mathcal{J}_{n}} \Omega^{0}(t)$. Therefore,

$$
\begin{aligned}
\left|\tilde{f}(x, t)-\tilde{f}\left(x, t_{n}\right)\right|= & \mid \max \{0, f(x, t)+\Delta \psi\} \\
& -\max \left\{0, f\left(x, t_{n}\right)+\Delta \psi\right\} \mid \\
\leq & \left|f(x, t)-f\left(x, t_{n}\right)\right| .
\end{aligned}
$$

Thus, inequality (4.9) follows.

Let $B_{n}^{2}=B_{n_{1}}^{2}+B_{n_{2}}^{2}$, where

$$
\begin{aligned}
& B_{n_{1}}^{2}=\frac{1}{\Delta t} \int_{\mathcal{J}_{n}}\left[\int_{\Omega \backslash D_{n}}\left(\tilde{f}(x, t)-\tilde{f}\left(x, t_{n}\right)\right) e^{n} d x\right] d t \\
& B_{n_{2}}^{2}=\frac{1}{\Delta t} \int_{\mathcal{J}_{n}}\left[\int_{D_{n}}\left(\tilde{f}(x, t)-\tilde{f}\left(x, t_{n}\right)\right) e^{n} d x\right] d t .
\end{aligned}
$$

Thus by using inequality (4.9), Hölder's inequality and Cauchy-Schwarz inequality, we get

$$
\left|B_{n_{1}}^{2}\right| \leq \frac{\epsilon}{2}\left\|e^{n}\right\|_{L^{2}(\Omega)}^{2}+\frac{1}{2 \epsilon}(\Delta t)\left\|\frac{\partial f}{\partial t}\right\|_{L^{2}\left(\mathcal{J}_{n}, L^{2}(\Omega)\right)}^{2} .
$$

Therefore, by the fact $N=\frac{T}{\Delta t}$, we have

$$
\sum_{n=1}^{N}\left|B_{n_{1}}^{2}\right| \Delta t \leq \frac{T \epsilon}{2} \max _{n \in\{1, \ldots, N\}}\left\|e^{n}\right\|_{L^{2}(\Omega)}^{2}+\frac{1}{2 \epsilon}(\Delta t)^{2}\left\|\frac{\partial f}{\partial t}\right\|_{L^{2}\left(\mathcal{J} ; L^{2}(\Omega)\right)}^{2} .
$$

On the other hand,

$$
\begin{aligned}
\left|B_{n_{2}}^{2}\right| & \leq \frac{1}{\Delta t} \int_{\mathcal{J}_{n}}\left[\int_{D_{n}}\left|\tilde{f}(x, t)-\tilde{f}\left(x, t_{n}\right)\right| e^{n} \mid d x\right] d t \\
& \leq \frac{2}{\Delta t}\left(\|f\|_{L^{\infty}\left(\mathcal{J}_{n}, L^{\infty}(\Omega)\right)}+\|\Delta \psi\|_{L^{\infty}\left(D_{n}\right)}\right) \int_{\mathcal{J}_{n}} \int_{D_{n}}\left|e^{n}\right| d x d t \\
& =2\left(\|f\|_{L^{\infty}\left(\mathcal{J}_{n}, L^{\infty}(\Omega)\right)}+\|\Delta \psi\|_{L^{\infty}\left(D_{n}\right)}\right) \int_{D_{n}}\left|e^{n}\right| d x
\end{aligned}
$$


By using Hölder's inequality, we have

$$
r_{n} \leq\left\|e^{n}\right\|_{L^{2}(\Omega)}\left(m\left(D_{n}\right)\right)^{\frac{1}{2}}, \text { where } r_{n}:=\int_{D_{n}}\left|e^{n}\right| d x
$$

On the other hand, by using Hölder's inequality with $\frac{1}{p}+\frac{1}{q}=1, p \geq 1$ and the fact, $\sup _{p \geq 1} \frac{1}{\sqrt{ } p}\|v\|_{L^{p}(\Omega)} \leq C\|v\|_{H^{1}(\Omega)}, v$ $\in H^{1}(\Omega)$ that in [31] , we deduce

$$
r_{n} \leq\left\|e^{n}\right\|_{L^{p}\left(D_{n}\right)}\left(m\left(D_{n}\right)\right)^{\frac{1}{q}} \leq C \sqrt{ } p\left\|e^{n}\right\|_{H^{1}(\Omega)}\left(m\left(D_{n}\right)\right)^{\frac{1}{q}} .
$$

Let $\left\{N_{1}, N_{2}\right\}$ be a partition of $\{1, \ldots, N\}$ into two disjoint subsets. Taking summation over $n \in\{1, \ldots, N\}$, then applying inequality (4.12) and (4.13) over $N_{1}$ and $N_{2}$ respectively, we obtain

$$
\sum_{n=1}^{N} r_{n} \Delta t \leq \sum_{n \in N_{1}}\left(m\left(D_{n}\right)\right)^{\frac{1}{2}}\left\|e^{n}\right\|_{L^{2}(\Omega)} \Delta t+C \sum_{n \in N_{2}} \sqrt{ } p\left(m\left(D_{n}\right)\right)^{\frac{1}{q}}\left\|e^{n}\right\|_{H^{1}(\Omega)} \Delta t .
$$

Using Cauchy-Schwarz inequality, we have

$$
\begin{aligned}
\sum_{n \in N_{1}}\left(m\left(D_{n}\right)\right)^{\frac{1}{2}}\left\|e^{n}\right\|_{L^{2}(\Omega)} \Delta t & \leq \max _{n \in N_{1}}\left\|e^{n}\right\|_{L^{2}(\Omega)} \sum_{n \in N_{1}}\left(m\left(D_{n}\right)\right)^{\frac{1}{2}} \Delta t \\
& \leq \max _{n \in\{1, \ldots, N\}}\left\|e^{n}\right\|_{L^{2}(\Omega)} \sum_{n \in N_{1}}\left(m\left(D_{n}\right)\right)^{\frac{1}{2}} \Delta t \\
& \leq \max _{n \in\{1, \ldots, N\}} \frac{\epsilon}{2}\left\|e^{n}\right\|_{L^{2}(\Omega)}^{2}+\frac{1}{2 \epsilon}\left(\sum_{n \in N_{1}}\left(m\left(D_{n}\right)\right)^{\frac{1}{2}} \Delta t\right)^{2} .
\end{aligned}
$$

In the last term of (4.14), using Cauchy-Schwarz inequality, we deduce

$$
\begin{aligned}
\sum_{n \in N_{2}} \sqrt{ } p\left(m\left(D_{n}\right)\right)^{\frac{1}{q}}\left\|e^{n}\right\|_{H^{1}(\Omega)} \Delta t \leq & \Delta t \sum_{n \in N_{2}}\left[\frac{\epsilon}{2}\left\|e^{n}\right\|_{H^{1}(\Omega)}^{2}+\frac{1}{2 \epsilon} p\left(m\left(D_{n}\right)\right)^{\frac{2}{q}}\right] \\
\leq & \frac{\epsilon}{2} \sum_{n=1}^{N}\left\|e^{n}\right\|_{H^{1}(\Omega)}^{2} \Delta t+\frac{1}{2 \epsilon} \Delta t \sum_{n \in N_{2}} p\left(m\left(D_{n}\right)\right)^{\frac{2}{q}} \\
\leq & \frac{\epsilon}{2} \max _{n \in\{1, \ldots, N\}}\left\|e^{n}\right\|_{L^{2}(\Omega)}^{2} N \cdot \Delta t+\frac{\epsilon}{2} \sum_{n=1}^{N} a\left(e^{n}, e^{n}\right) \Delta t \\
& +\frac{1}{2 \epsilon} \Delta t \sum_{n \in N_{2}} p\left(m\left(D_{n}\right)\right)^{\frac{2}{q}} .
\end{aligned}
$$

Here, we define $\mathrm{F}$ as the following

$$
F:=C(\Delta t)^{2}\left[\left(\sum_{n \in N_{1}}\left(m\left(D_{n}\right)\right)^{\frac{1}{2}}\right)^{2}+p(\Delta t)^{-1} \sum_{n \in N_{2}}\left(m\left(D_{n}\right)\right)^{\frac{2}{q}}\right] .
$$

By using [1, Lemma 3] with assumption (3.3), we have

$$
F \leq C(\Delta t)^{\frac{3}{2}}\left(\log \frac{1}{\Delta t}\right)^{\frac{1}{2}} .
$$

Thus, by above estimation of $\mathrm{F}$ and from (4.14), (4.15) and (4.16), we have

$$
\sum_{n=1}^{N} r_{n} \Delta t \leq \epsilon \max _{n \in\{1, \ldots, N\}}\left\|e^{n}\right\|_{L^{2}(\Omega)}^{2}+\epsilon \sum_{n=1}^{N} a\left(e^{n}, e^{n}\right) \Delta t+C(\Delta t)^{\frac{3}{2}}\left(\log \frac{1}{\Delta t}\right)^{\frac{1}{2}} .
$$

Using the fact $f \in C\left(\mathcal{J} ; L^{\infty}(\Omega)\right)$ in (4.11), and from (4.17), we have

$$
\sum_{n=1}^{N}\left|B_{n_{2}}^{2}\right| \Delta t \leq C \sum_{n=1}^{N} r_{n} \Delta t
$$


Thus by using above inequality, and from (4.10) and (4.17), we have

$$
\begin{array}{r}
\sum_{n=1}^{N}\left|B_{n}^{2}\right| \Delta t \leq C \epsilon \max _{n \in\{1, \ldots, N\}}\left\|e^{n}\right\|_{L^{2}(\Omega)}^{2}+\frac{1}{2 \epsilon}(\Delta t)^{2}\left\|\frac{\partial f}{\partial t}\right\|_{L^{2}\left(\mathcal{J} ; L^{2}(\Omega)\right)}^{2} \\
+C \epsilon \sum_{n=1}^{n} a\left(e^{n}, e^{n}\right) \Delta t \\
+C(\Delta t)^{\frac{3}{2}}\left(\log \frac{1}{\Delta t}\right)^{\frac{1}{2}} .
\end{array}
$$

Finally, from definition of $E_{4}$, (4.7) and by using (4.8), (4.18) and

$$
\sum_{n=1}^{N}\left\|e^{n}\right\|_{H^{1}(\Omega)}^{2} \Delta t \leq T \max _{n \in\{1, \ldots, N\}}\left\|e^{n}\right\|_{L^{2}(\Omega)}^{2}+\sum_{n=1}^{N} a\left(e^{n}, e^{n}\right) \Delta t
$$

we deduce

$$
\begin{aligned}
E_{4} \leq & \sum_{n=1}^{N}\left|B_{n}^{1}\right| \Delta t+\sum_{n=1}^{N}\left|B_{n}^{2}\right| \Delta t \\
\leq & C \epsilon \max _{n \in\{1, \ldots, N\}}\left\|e^{n}\right\|_{L^{2}(\Omega)}^{2}+C \epsilon \sum_{n=1}^{N} a\left(e^{n}, e^{n}\right) \Delta t \\
& +T \epsilon \max _{n \in\{1, \ldots, N\}}\left\|W_{h}^{n}-\tilde{W}_{h}^{n}\right\|_{H^{1}(\Omega)}^{2} \\
& +2 T\|\Delta w\|_{L^{\infty}\left(\mathcal{J} ; L^{2}(\Omega)\right)} \max _{n \in\{1, \ldots, N\}}\left\|W_{h}^{n}-\tilde{W}_{h}^{n}\right\|_{L^{2}(\Omega)} \\
& +\frac{1}{2 \epsilon}(\Delta t)^{2}\left[\left\|\frac{\partial w}{\partial t}\right\|_{L^{2}\left(\mathcal{J} ; H^{1}(\Omega)\right)}^{2}+\left\|\frac{\partial f}{\partial t}\right\|_{L^{2}\left(\mathcal{J} ; L^{2}(\Omega)\right)}^{2}\right]+C(\Delta t)^{\frac{3}{2}}\left(\log \frac{1}{\Delta t}\right)^{\frac{1}{2}} .
\end{aligned}
$$

\section{Estimation of $E_{5}$ :}

$$
\begin{aligned}
E_{5}=\sum_{n=1}^{N} A_{n}^{5} \Delta t, \text { where } A_{n}^{5}= & \left(\frac{\partial^{+} w}{\partial t}\left(t_{n}\right), \tilde{W}_{h}^{n}-W_{h}^{n}\right) \\
= & \int_{\Omega^{+}\left(t_{n}\right)}\left(\Delta w\left(t_{n}\right)+f^{n}+\Delta \psi(x)\right)\left(\tilde{W}_{h}^{n}\right. \\
& \left.-W_{h}^{n}\right) d x+\int_{\Omega^{0}\left(t_{n}\right)} \max \left\{0, f^{n}+\Delta \psi(x)\right\}\left(\tilde{W}_{h}^{n}-W_{h}^{n}\right) d x .
\end{aligned}
$$

By using Hölder's inequality and triangle inequality with $N=\frac{T}{\Delta t}$, we have the following.

$$
\begin{aligned}
E_{5}=\sum_{n=1}^{N} A_{n}^{5} \Delta t \leq & T\left[\|\Delta w\|_{L^{\infty}\left(\mathcal{J} ; L^{2}(\Omega)\right)}+2\|f\|_{L^{\infty}\left(\mathcal{J} ; L^{2}(\Omega)\right)}\right. \\
& \left.+2\|\Delta \psi\|_{L^{2}(\Omega)}\right] \max _{n \in\{1, \ldots, N\}}\left\|\tilde{W}_{h}^{n}-W_{h}^{n}\right\|_{L^{2}(\Omega)} .
\end{aligned}
$$

Estimation of $E_{6}: E_{6}=\sum_{n=1}^{N} A_{n}^{6} \Delta t$, where $A_{n}^{6}=-\left(\partial w^{n}, \eta^{n}\right) \leq\left\|\partial w^{n}\right\|_{L^{2}(\Omega)} \quad\left\|\eta^{n}\right\|_{L^{2}(\Omega)}$, and from definition of $\partial w^{n}$ we have $\left\|\partial w^{n}\right\|_{L^{2}(\Omega)}=\frac{1}{\Delta t}\left(\int_{\Omega}\left|\int_{t_{n-1}}^{t_{n}} \frac{\partial w}{\partial t}(s) d s\right|^{2} d x\right)^{\frac{1}{2}}$. Since $\frac{\partial w}{\partial t} \in L^{\infty}\left(\mathcal{J} ; L^{\infty}(\Omega)\right)$, there exists $C>0$ such that $\left|\frac{\partial w}{\partial t}\right| \leq \sup _{t \in \mathcal{J}}$ ess $\sup _{x \in \Omega}\left|\frac{\partial w}{\partial t}\right| \leq C$ a.e. Thus,

$$
\begin{aligned}
& \int_{t_{n-1}}^{t_{n}}\left|\frac{\partial w}{\partial t}\right| d s \leq C \Delta t \\
\Rightarrow & \left(\left|\int_{t_{n-1}}^{t_{n}}\right| \frac{\partial w}{\partial t}|d s|\right)^{2} \leq C^{2}(\Delta t)^{2} \\
\Rightarrow & \int_{\Omega}\left(\left|\int_{t_{n-1}}^{t_{n}}\right| \frac{\partial w}{\partial t}|d s|\right)^{2} \leq C^{2}(\Delta t)^{2} m(\Omega) \\
\Rightarrow & \left(\int_{\Omega}\left(\left|\int_{t_{n-1}}^{t_{n}}\right| \frac{\partial w}{\partial t}|d s|\right)^{2}\right)^{\frac{1}{2}} \leq C(\Delta t)(m(\Omega))^{\frac{1}{2}} .
\end{aligned}
$$


Therefore, $\left\|\partial w^{n}\right\|_{L^{2}(\Omega)} \leq C(m(\Omega))^{\frac{1}{2}}$, and hence

$$
\begin{aligned}
\sum_{n=1}^{N}\left|A_{n}^{6}\right| \Delta t & \leq C(m(\Omega))^{\frac{1}{2}} \max _{n \in\{1, \ldots, N\}}\left\|\eta^{n}\right\|_{L^{2}(\Omega)} \sum_{n=1}^{N} \Delta t \\
& \leq C h^{2}\|w\|_{L^{\infty}\left(\mathcal{J} ; H^{2}(\Omega)\right)} .
\end{aligned}
$$

This implies, $E_{6} \leq C h^{2}\|w\|_{L^{\infty}\left(\mathcal{J} ; H^{2}(\Omega)\right)}$.

From Corollary 3.4, and the estimation of $E_{4}$ and $E_{5}$ we have following.

and $E_{5} \leq C h^{2}$.

$$
\begin{aligned}
E_{4} \leq & C \epsilon \max _{n \in\{1, \ldots, N\}}\left\|e^{n}\right\|_{L^{2}(\Omega)}^{2}+C \epsilon \sum_{n=1}^{N} a\left(e^{n}, e^{n}\right) \Delta t \\
& +C h^{2}+C(\Delta t)^{2}+C(\Delta t)^{\frac{3}{2}}\left(\log \frac{1}{\Delta t}\right)^{\frac{1}{2}},
\end{aligned}
$$

By making $\epsilon$ small, from the above estimations of $E_{i}, i=1, \ldots, 6$ and (4.5), there exists a constant $C>0$ independent on $\Delta t$ and $h$ such that,

$$
\begin{aligned}
& \max _{n \in\{1, \ldots, N\}}\left\|e^{n}\right\|_{L^{2}(\Omega)}^{2}+\sum_{n=1}^{N} a\left(e^{n}, e^{n}\right) \Delta t \leq C\left\|e^{0}\right\|_{L^{2}(\Omega)}^{2}+C h^{2}+C(\Delta t)^{2}+C(\Delta t)^{\frac{3}{2}}\left(\log \frac{1}{\Delta t}\right)^{\frac{1}{2}} . \\
& \text { Therefore, } \max _{n \in\{1, \ldots, N\}}\left\|e^{n}\right\|_{L^{2}(\Omega)}+\left(\sum_{n=1}^{N}\left\|e^{n}\right\|_{H^{1}(\Omega)}^{2} \Delta t\right)^{\frac{1}{2}} \leq C\left[h+(\Delta t)^{\frac{3}{4}}\left(\log \frac{1}{\Delta t}\right)^{\frac{1}{4}}\right] .
\end{aligned}
$$

These complete the proof.

Remark 4.2. We observe that if $\frac{\partial^{2} u}{\partial t^{2}} \in L^{2}\left(\mathcal{J} ; H^{-1}(\Omega)\right)$ then we get $\Delta t$ in place of $(\Delta t)^{\frac{3}{4}}\left(\log \frac{1}{\Delta t}\right)^{\frac{1}{4}}$ in the above error estimation (cf. [32]). For an example, if the variational inequality originates from a Stefan problem then $\frac{\partial u}{\partial t} \in C(\mathcal{J} ; C(\bar{\Omega}))$ and $\frac{\partial^{2} u}{\partial t^{2}} \in L^{2}\left(\mathcal{J} ; L^{2}(\Omega)\right)$, and in this case we get $\Delta t$ in place of $(\Delta t)^{\frac{3}{4}}\left(\log \frac{1}{\Delta t}\right)^{\frac{1}{4}}$. But in general it is not possible to get more global regularity of the solution $u$ than that given by (2.3) even if the given data is smooth.

\section{Numerical experiments}

In this section, we present numerical experiments to illustrate the theoretical results derived in the previous section. By considering $2 d$ oscillating moving circle from [5], as a model example, we test the validity of the a priori error estimates derived in Theorem 4.1.

\subsection{2d oscillating moving circle}

Let $\Omega$ be the square $(-1,1) \times(-1,1), \mathcal{J}=[0,0.25]$ be the time interval, and let the non-contact and contact sets be

$$
\Omega^{+}(t):=\left\{(x, y) \in \Omega: r(t)>r_{0}(t)\right\} \text { and } \Omega^{0}(t):=\left\{(x, y) \in \Omega: r(t) \leq r_{0}(t)\right\}
$$

respectively, where $r(t)=\left\{\left(x-r_{1} \cos (a \pi t)\right)^{2}+\left(y-r_{1} \sin (a \pi t)\right)^{2}\right\}^{\frac{1}{2}}, r_{0}(t)=\frac{1}{3}+0.3 \sin (4 a \pi t)$, $r_{1}=\frac{1}{3}$ and $a=4$. The exact solution $w$ is

$$
w(x, y, t)=\left\{\begin{array}{lll}
\frac{1}{2}\left(r^{2}(t)-r_{0}^{2}(t)\right)^{2} & \text { if } \quad(x, y) \in \Omega^{+}(t) \\
0 & \text { if } \quad(x, y) \in \Omega^{0}(t) .
\end{array}\right.
$$

The initial and boundary conditions are given by $w$. The obstacle is $\psi:=0$ and forcing function $f$ is

$$
f(x, y, t)= \begin{cases}4\left(r_{0}^{2}(t)-2 r^{2}(t)-\frac{1}{2}\left(r^{2}(t)-r_{0}^{2}(t)\right)\left(p(t)+r_{0}(t) r_{0}^{\prime}(t)\right)\right) & \text { if } \quad(x, y) \in \Omega^{+}(t), \\ -4 r_{0}^{2}(t)\left(1-r^{2}(t)+r_{0}^{2}(t)\right) & \text { if } \quad(x, y) \in \Omega^{0}(t),\end{cases}
$$

where $p(t)=\left(x-c_{1}(t)\right) c_{1}^{\prime}(t)+\left(y-c_{2}(t)\right) c_{2}^{\prime}(t), c_{1}(t)=r_{1} \cos (a \pi t)$ and $c_{2}(t)=r_{1} \sin (a \pi t)$. The free boundary is an 
Table 5.1

Errors and orders of convergence for $h$.

\begin{tabular}{rlll}
\hline$N$ & $h$ & Errcom & Order \\
\hline 10 & $2 / 4$ & 1.50425057 & - \\
40 & $2 / 8$ & 0.53532046 & 1.49057020 \\
160 & $2 / 16$ & 0.19618411 & 1.44819457 \\
640 & $2 / 32$ & 0.08112544 & 1.27398188 \\
2560 & $2 / 64$ & 0.03635862 & 1.15785670 \\
\hline
\end{tabular}

Table 5.2

Errors and orders of convergence for $\Delta t$.

\begin{tabular}{rlll}
\hline$N$ & $h$ & Errcom & Order \\
\hline 5 & $2 / 4$ & 1.63721581 & - \\
10 & $2 / 8$ & 0.90592722 & 0.85377744 \\
20 & $2 / 16$ & 0.51329971 & 0.81959369 \\
40 & $2 / 32$ & 0.25140404 & 1.02979362 \\
80 & $2 / 64$ & 0.12514001 & 1.00646467 \\
160 & $2 / 128$ & 0.063533927 & 0.97794401 \\
\hline
\end{tabular}

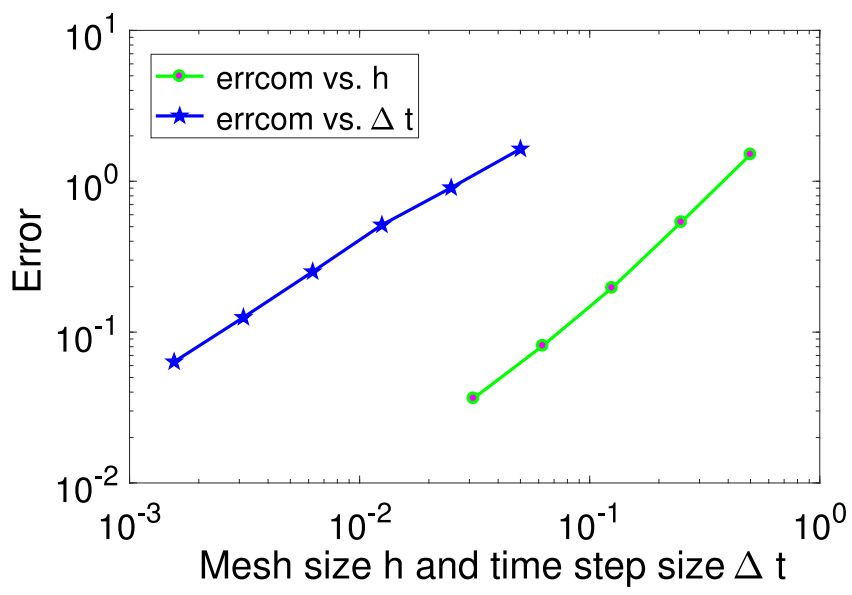

Fig. 5.1. Time step size $\Delta t$ and errcom.

oscillating circle with radius $r_{0}(t)$ and center $\left(c_{1}(t), c_{2}(t)\right)$ moving anticlockwise along the circle of radius $r_{1}$ centered at the origin.

In Table 5.1, we have shown order of convergence in the norm defined below for space variable $h$ with $\Delta t=\mathcal{O}\left(h^{2}\right)$. Define

$$
\begin{aligned}
\text { errcom } & =\max _{n \in\{1, \ldots, N\}}\left\|e^{n}\right\|_{L^{2}(\Omega)}^{2}+\sum_{n=1}^{N} a\left(e^{n}, e^{n}\right) \Delta t, \\
N & =\text { number of time steps. }
\end{aligned}
$$

In Table 5.2, we have displayed order of convergence for time variable $\Delta t$ with $\Delta t=\mathcal{O}(h)$ with the same error norm as in above. We expect that the convergence rate with uniform time-step would be about $\mathcal{O}\left(\Delta t^{\frac{3}{4}}\right)$ and for space $\mathcal{O}(h)$ with uniform mesh refinement. Our experiments attend this expectation (see Figs. 5.1 and 5.2).

\section{Acknowledgment}

Both the authors acknowledge the financial support from the UGC center for advanced study. 

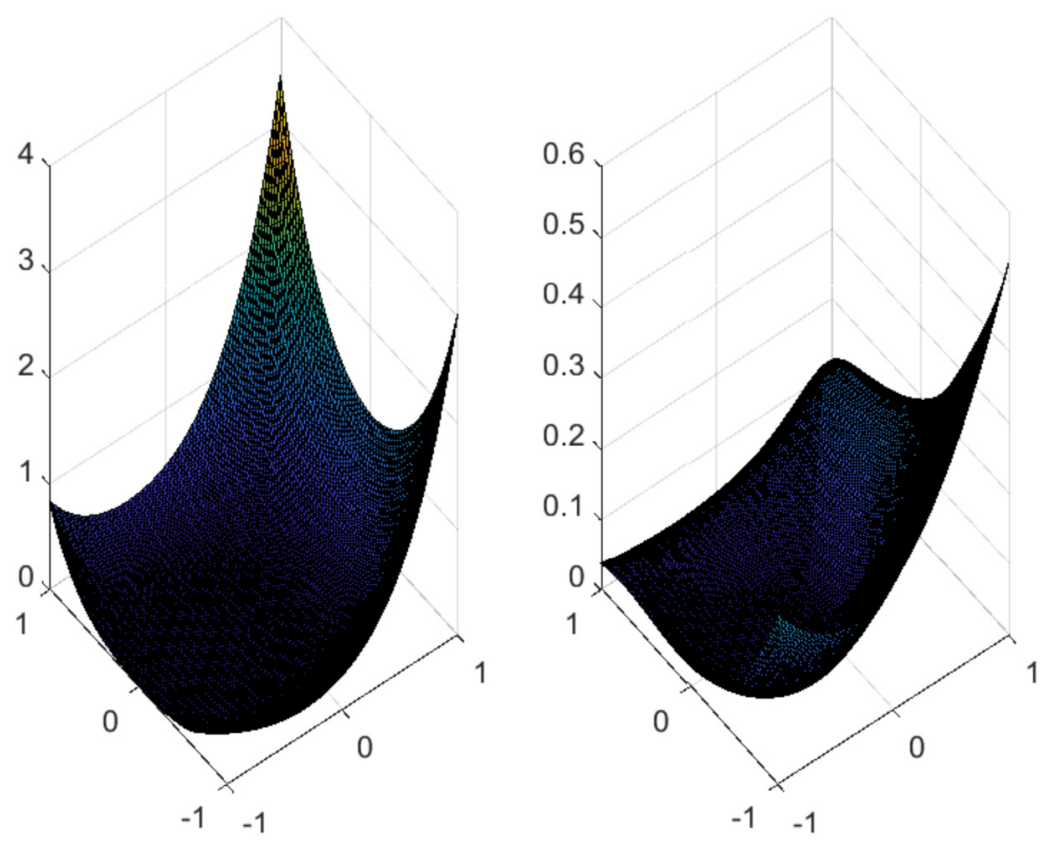

Fig. 5.2. The computed (left) and error $w-W_{h}$ (right) at time $t=0.25$.

\section{References}

[1] C. Johnson, A convergence estimate for an approximation of a parabolic variational inequality, SIAM J. Numer. Anal. 13 (1976) $599-606$.

[2] A.E. Berger, R.S. Falk, An error estimate for the truncation method for the solution of parabolic obstacle variational inequalities, Math. Comp. 31 (1977) 619-628.

[3] A. Fetter, $L^{\infty}$-error estimate for an approximation of a parabolic variational inequality, Numer. Math. 50 (1987) 557-565.

[4] C. Vuik, An $L^{2}$-error estimate for an approximation of the solution of a parabolic variational inequality, Numer. Math. 57 (1990) $453-471$.

[5] K.S. Moon, R.H. Nochetto, T. von Petersdorff, C.S. Zhang, A posteriori error analysis for parabolic variational inequalities, M2AN Math. Model. Numer. Anal. 41 (3) (2007) 485-511.

[6] X. Yang, G. Wang, X. Gu, Numerical solution for a parabolic obstacle problem with nonsmooth initial data, Numer. Methods Partial Differential Equations 30 (5) (2014) 1740-1754.

[7] M.A. Bencheikh le Hocine, S. Boulaaras, M. Haiour, An optimal $L^{\infty}$-error estimate for an approximation of a parabolic variational inequality, Numer. Funct. Anal. Optim. 37 (1) (2016) 1-18.

[8] E. Otárola, J.A. Salgado, Finite element approximation of the parabolic fractional obstacle problem, SIAM J. Numer. Anal. 54 (4) (2016) $2619-2639$.

[9] W. Allegretto, Y. Lin, H. Yang, Finite element error estimates for a nonlocal problem in american option valuation, SIAM J. Numer. Anal. 39 (3) (2001) 834-857.

[10] R. Company, V.N. Egorova, L. Jódar, C. Vázquez, Finite difference methods for pricing american put option with rationality parameter: numerical analysis and computing, J. Comput. Appl. Math. 304 (2016) 1-17.

[11] S. Jo, M. Yang, G. Kim, On convergence of Laplace inversion for the American put option under the CEV model, J. Comput. Appl. Math. 305 (2016) 36-43.

[12] A.M. Matache, P.A. Nitsche, C. Schwab, Wavelet Galerkin pricing of american options on Lévy driven assets, Quant. Finance 5 (4) (2005) $403-424$.

[13] A.K. Pani, P.C. Das, A priori error estimates for a single-phase quasilinear Stefan problem in one space dimension, IMA J. Numer. Anal. 11 (1991) 377-392.

[14] G. Wang, X. Yang, The regularization method for a degenerate parabolic variational inequality arising from American option valuation, Int. J. Numer. Anal. Model. 5 (2) (2008) 222-238.

[15] Y. Zhang, Error estimates for the numerical approximation of time-dependent flow of Bingham fluid in cylindrical pipes by the regularization method, Numer. Math. 96 (1) (2003) 153-184.

[16] S.C. Brenner, L.R. Scott, The Mathematical Theory of Finite Element Methods, third ed., Springer-Verlag, New York, 2008.

[17] P.G. Ciarlet, The Finite Element Method for Elliptic Problems, North-Holland, Amsterdam, 1978.

[18] R. Glowinski, Numerical Methods for Nonlinear Variational Problems, Springer-Verlag, Berlin, 2008.

[19] S. Kesavan, Topics in Functional Analysis and Applications, New Age International Ltd, Publishers, New Delhi, 1989.

[20] D. Kinderlehrer, G. Stampacchia, An Introduction to Variational Inequalities and their Applications, SIAM, Philadelphia, 2000.

[21] H.L. Royden, Real Analysis, third ed., Prnetice-Hall of India Pvt. Ltd, New Delhi, 2008.

[22] F.T. Suttmeier, Numerical solution of Variational Inequalities by Adaptive Finite Elements, Vieweg+Teubner Research, 2008.

[23] V. Thomée, Galerkin Finite Element Methods for Parabolic Problems, second ed., in: Springer Series in Computational Mathematics, vol. 25, Springer, Berlin, 2006.

[24] R.S. Falk, Error estimates for the approximation of a class of variational inequalities, Math. Comp. 28 (1974) 963-971.

[25] M. Hintermüller, K. Ito, K. Kunish, The primal-dual active set strategy as a semismooth Newton method, SIAM J. Optim. 13 (2003) $865-888$.

[26] R.H. Nochetto, G. Savaré, C. Verdi, A posteriori error estimates for variable time-step discretizations of nonlinear evolution equations, Comm. Pure Appl. Math. 53 (5) (2000) 525-589.

[27] Z. Chen, R. Nochetto, Residual type a posteriori error estimates for elliptic obstacle problems, Numer. Math. 84 (2000) $527-548$. 
[28] P. Hild, S. Nicaise, A posteriori error estimates of residual type for Signorini's problem, Numer. Math. 101 (2005) 523-549.

[29] H. Brezis, Problèmes unilatéraux, J. Math. Pures Appl. 57 (1972) 1-168.

[30] S. Gaddam, T. Gudi, Inhomogeneous Dirichlet boundary condition in the a posteriori error control of the obstacle problem, Comput. Math. Appl. 75 (7) (2018) 2311-2327, http://dx.doi.org/10.1016/j.camwa.2017.12.010, (Published online on 28 2017).

[31] J. Peetre, Espace d'interpolation et théorème de soboleff, Ann. Inst. Fourier (Grenoble) 16 (1) (1966) 279-317.

[32] T. Dupont, Some $L^{2}$ error estimates for parabolic Galerkin methods, in: A.K. Aziz (Ed.), The Mathematical Foundation of the Finite Element Method with Applications to Partial Differential Equations, Academic Press, New York, 1972. 